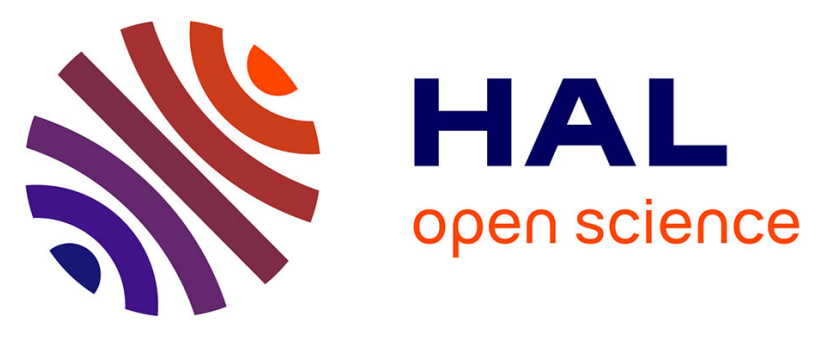

\title{
Residence time distributions for hydrologic systems: Mechanistic foundations and steady-state analytical solutions
}

\author{
Sarah Leray, Nicholas B. Engdahl, Arash Massoudieh, Etienne Bresciani, \\ James Mccallum
}

\section{To cite this version:}

Sarah Leray, Nicholas B. Engdahl, Arash Massoudieh, Etienne Bresciani, James Mccallum. Residence time distributions for hydrologic systems: Mechanistic foundations and steady-state analytical solutions. Journal of Hydrology, 2016, RESIDENCE TIMES IN SUBSURFACE HYDROLOGICAL SYSTEMS: Signature of hydrological processes and impact on environmental applications, 543 (Part A), pp.67-87. 10.1016/j.jhydrol.2016.01.068 . insu-01271309

\section{HAL Id: insu-01271309 \\ https://hal-insu.archives-ouvertes.fr/insu-01271309}

Submitted on 9 Feb 2016

HAL is a multi-disciplinary open access archive for the deposit and dissemination of scientific research documents, whether they are published or not. The documents may come from teaching and research institutions in France or abroad, or from public or private research centers.
L'archive ouverte pluridisciplinaire HAL, est destinée au dépôt et à la diffusion de documents scientifiques de niveau recherche, publiés ou non, émanant des établissements d'enseignement et de recherche français ou étrangers, des laboratoires publics ou privés. 


\section{Accepted Manuscript}

Residence time distributions for hydrologic systems: Mechanistic foundations and steady-state analytical solutions

Sarah Leray, Nicholas B. Engdahl, Arash Massoudieh, Etienne Bresciani, James Mccallum

PII: S0022-1694(16)30008-7

DOI: http://dx.doi.org/10.1016/j.jhydrol.2016.01.068

Reference: HYDROL 21027

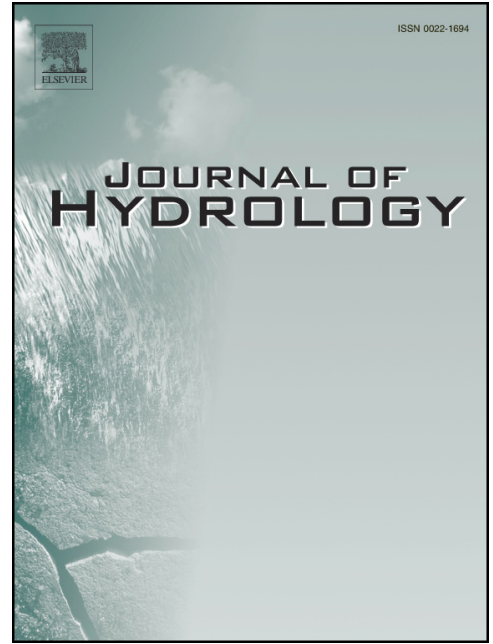

To appear in:

Journal of Hydrology

Please cite this article as: Leray, S., Engdahl, N.B., Massoudieh, A., Bresciani, E., Mccallum, J., Residence time distributions for hydrologic systems: Mechanistic foundations and steady-state analytical solutions, Journal of Hydrology (2016), doi: http://dx.doi.org/10.1016/j.jhydrol.2016.01.068

This is a PDF file of an unedited manuscript that has been accepted for publication. As a service to our customers we are providing this early version of the manuscript. The manuscript will undergo copyediting, typesetting, and review of the resulting proof before it is published in its final form. Please note that during the production process errors may be discovered which could affect the content, and all legal disclaimers that apply to the journal pertain. 


\title{
Residence time distributions for
}

\section{hydrologic systems: Mechanistic}

\section{foundations and steady-state analytical}

\section{solutions}

Sarah LERAY ${ }^{1}$, Nicholas B. ENGDAHL ${ }^{2}$, Arash MASSOUDIEH ${ }^{3}$, Etienne BRESCIANI $^{4}$, James McCALLUM ${ }^{4}$

${ }^{1}$ Géosciences Rennes (UMR 6118 CNRS), Université de Rennes 1, Rennes, France.

2 Department of Civil and Environmental Engineering, Washington State University, Pullman, WA, USA.

${ }^{3}$ Department of Civil Engineering, Catholic University of America, Washington, D. C., USA.

${ }^{4}$ School of the Environment / National Centre for Groundwater Research and Training, Flinders University, Adelaide, SA, Australia.

\begin{abstract}
This review presents the physical mechanisms generating residence time distributions (RTDs) in hydrologic systems with a focus on steady-state analytical solutions. Steadystate approximations of the RTD in hydrologic systems have seen widespread use over the last half-century because they provide a convenient, simplified modeling framework for a wide range of problems. The concept of an RTD is useful anytime that characterization of the timescales of flow and transport in hydrologic systems is important, which includes topics like water quality, water resource management,
\end{abstract}


contaminant transport, and ecosystem preservation. Analytical solutions are often adopted as a model of the RTD and a broad spectrum of models from many disciplines has been applied. Although these solutions are typically reduced in dimensionality and limited in complexity, their ease of use makes them preferred tools, specifically for the interpretation of tracer data. Our review begins with the mechanistic basis for the governing equations, highlighting the physics for generating a RTD, and a catalog of analytical solutions follows. This catalog explains the geometry, boundary conditions and physical aspects of the hydrologic systems, as well as the sampling conditions, that altogether give rise to specific RTDs. The similarities between models are noted, as are the appropriate conditions for their applicability. The presentation of simple solutions is followed by a presentation of more complicated analytical models for RTDs, including serial and parallel combinations, lagged systems, and non-Fickian models. The conditions for the appropriate use of analytical solutions are discussed, and we close with some thoughts on potential applications, alternative approaches, and future directions for modeling hydrologic residence time.

\section{Introduction}

Residence time is one of the most general, widespread concepts in all of hydrology. This generality stems from the fact that, regardless of any specific system being considered (watershed, lake, ocean, etc...), water is moving and cycling into and out of neighboring systems, and the amount of time spent in any section of the connected network is an important consideration for many problems. Residence time has application to water quality, risk assessment, contaminant remediation, characterization, habitat restoration, toxicity, reaction rates, age dating, turnover times in lakes, and ocean circulation, amongst others (Cirpka and Kitanidis, 2001; Delhez et al., 1999; Maxwell et al., 2003; Neumann et al., 2008; Seeboonruang and Ginn, 2006; Solomon et al., 2010). Despite this wide range of applications, the principles of residence time are fundamentally the same in that they are all concerned with the amount of time water, or some element transported by it, has spent in the system. 
The common mechanistic framework that unifies residence time theory in hydrology is often masked by the terminology and assumptions adopted for a particular study or application. This raises confusion since two studies on residence time may be referring to something altogether different. The definition adopted here will be general, but robust: the residence time is defined as the amount of time a moving element has spent in a hydrologic system, which is typically the water mass but could be solutes. Alternative names for residence time include transit time, travel time, age, and exposure time (Ali et al., 2014; Beven, 2010; Campana, 1987; Ginn, 1999; Gomez and Wilson, 2013; McDonnell et al., 2010; Schwientek et al., 2009). The latter is strictly the most general, but we will consider all of these as equivalent, at least mechanistically, for our discussion of residence time. For any finite volume of water (e.g. a water sample), a single residence time cannot be defined since the sample is composed of a mixture of water and this introduces the concept of a residence time distribution (RTD) (Bethke and Johnson, 2008), which will be the focus of this article.

The geometry, boundary conditions, and physical aspects of hydrologic systems cause RTDs to take on unique shapes that reflect the processes occurring within that system. A variety of solutions to the governing equations have been developed over the years for a range of systems including batch reactors, oceanic systems, aquifers, etc. To name but one, the most popular example is probably the so-called "exponential" model (Benettin et al., 2013; Danckwerts, 1953; Delhez et al., 1999; Luo and Cirpka, 2008). The simplest, and most common, of these solutions arise from the assumption of a steady-state system with respect to time. Many of these solutions are further simplified in dimensionality, which could mean assuming a 1-D model for a 3-D system and disregarding system's heterogeneity. All of these assumptions and simplifications can be constraining to such an extent that these analytical solutions might seem inapplicable or unsuitable. Still, they constitute a physical framework to understand what and how generic features, such as geometry or boundary conditions, generate the RTD. They provide a link between observations and system's characteristics that can help in testing quickly different conceptual representations and help in understanding why real hydrologic systems deviate from reference simple ones (Eberts et al., 2012; Leray et al., 2012). Another 
advantage of simple analytical models is that they are often formulated with a few parameters only. This allows for a straightforward characterization of the system with a small amount of data, hence offering an appealing approach to get a first approximation of hydrological processes even in data poor areas. In contrast, distributed models are more complicated to develop and require much more data to be fully characterized but offer a much greater flexibility for representing heterogeneity and unsteady conditions of a field situation. Both approaches (analytical and distributed models) are in fact complimentary.

The risk is high with analytical RTDs to take them as black box models of which parameters can be easily calibrated while the formal conditions and assumptions required to use them are overlooked, or are not clearly stated, causing confusion about the choice of a particular RTD model and about the consequences of this choice. In order to help in avoiding this, the intent of this paper is to expose clearly the physics behind the various models that are available for modeling steady-state RTDs analytically. To achieve this, this paper reviews and classifies available analytical solutions according to physical processes. This strategy is distinct from previous reviews on this topic (Małoszewski and Zuber, 1982; McGuire and McDonnell, 2006; Raats, 1974; Turner and Barnes, 1998), which presented mathematical models of RTDs (e.g. the exponential model or the linear model) and subsequent discussion of possible physical interpretations. In no way should these past reviews be viewed as incorrect but their presentation seems backwards to us for two reasons. First, it creates confusion regarding the use of mathematical models that can have different physical interpretations. For example, the exponential model is often associated with "perfect mixing" but exponential behavior can also emerge from other mechanisms such as the sampling of different flow paths. Second, it can be unclear whether a mathematical model selected for an RTD has any physical basis or whether it is selected simply because it provides a reasonably good fit; our aim is to assist readers in avoiding the latter whenever possible. The focal point of the paper is not the RTDs in themselves whose expressions are mostly well-known but the physical and operational (sampling) conditions, and their interplay for generating a RTD. The aim is to give clues 
for better understanding and modeling of the RTDs of real hydrologic systems. The introduction of additional little known solutions helps in that approach.

This paper focuses on steady-state solutions of RTD. While some of the limitations related to the steady-state assumption are discussed in section 5, a companion paper fully addresses the topic of transient RTDs which have received more attention in the recent years (Duffy, 2010; McDonnell and Beven, 2014; Rinaldo et al., 2015). The article is organized into three main sections, each of which is designed to be useful on its own. Section 2 is a detailed overview of the mechanistic basis for the governing equations and their theoretical development. The different derivation techniques are described as well as the basic solution techniques of the differential equations. Section 3 describes the origins of the commonly applied analytical solutions. The assumptions and conditions for these solutions are detailed with a focus on their applications to real-world scenarios. Both similarities and differences in concept between different analytical RTDs are highlighted. Emphasis is also placed on the kind of physical systems where these solutions are reasonable approximations. Section 4 discusses some of the more complicated analytical models for residence time including serial and parallel mixing models, lagged systems, and non-Fickian models. This section revises some of the earlier central assumptions (section 2) and explains why more complicated models may be required. Lastly, a broad discussion of potential applications is provided (section 5). Alternatives to physicallybased RTDs are also presented there: they are based on more abstract concepts and introduced as complementary approaches. All of these sections are intended to be somewhat independent of each other. For instance, those readers that are only interested in applying a specific model for residence time may wish to skip section 2 since sufficient detail about each model and its intended use are given in section 3, and so forth. References to specific applications of each kind of model are included but we do not discuss any of the applied studies in detail. Instead, we focus on explaining the mechanistic origin and utility of the different models and leave it to the reader to critique the validity of individual studies. 


\section{Governing equations and generic properties of RTDs}

The question of residence time in hydrologic systems always reduces to a mass balance. The difference between what follows and classical approaches for groundwater is that the mass will be formally distributed over an additional dimension of the problem space, creating the RTD. Eulerian and Lagrangian methods can be used to construct the appropriate mass balance statements but there are a few preliminary remarks that should be mentioned first.

The most precise accounting of mass possible is at the molecular level, where each water molecule has a Dirac delta distribution of mass and residence time. Obviously this is not a practical approach for hydrologic problems, but it is a useful conceptual starting point. The residence time is defined as the time since that molecule entered the hydrologic system up to the observation time. Physical processes may move the molecule but its mass is constant and its residence time is always Dirac delta distributed, linearly increasing over time. Addition of a second molecule to a sample creates the possibility for a non-uniform RTD so any sample of water is distributed over residence time whether it is a single molecule or all the water on the planet. Any difference in the residence time of the molecules creates a distribution that is no longer a Dirac delta and the more water molecules are sampled, the broader and more complex the RTD is expected to be (Figure 1). The goal of this section is to present the governing equations for the aqueous phase mass balance, distributed over residence time, for general conditions. This does not require one specific equation for flow to be selected, such as Darcy's law or Richard's equation, so it is completely general and cross-disciplinary. Our only assumption is that water is present and that it may move.

The fundamental statement of mass conservation is the continuity equation. Allowing for three spatial dimensions $\left(x_{1}, x_{2}, x_{3}\right)$, water in every point is also distributed over residence time which can therefore be defined as a fourth dimension. Allowing for a temporal dimension as well, a 5-D continuity equation can be derived for the aqueous mass density:

Steady-state analytical RTDs 


$$
\frac{\partial \rho_{w}}{\partial t}+\frac{\partial \rho_{w}}{\partial a}+\nabla \cdot \mathbf{j}_{w}\left(\rho_{w}\right)=0
$$

where $\rho_{w}$ is the aqueous phase mass density in the augmented space $\left(x_{1}, x_{2}, x_{3}, a\right), a$ is residence time, $t$ is time and $\mathbf{j}_{w}\left(\rho_{w}\right)$ is the total mass density flux (Cornaton and Perrochet, 2006; Ginn, 1999). At a given point in physical space $\left(x_{1}, x_{2}, x_{3}\right)$ and time, $\rho_{w}$ describes how the water mass is distributed over residence time. Normalizing $\rho_{w}$ by its integral over all possible residence times (i.e. by the classical density as defined in the physical space) gives the RTD, for which the integral is always one. Since mass is conserved, this equation must apply to any arbitrary material volume but is equally valid for fixed or moving reference frames. Alternate forms may include source/sink or reaction terms that alter the amount of mass within the material volume. Equation (1) is unique because it allows changes in $t$ and $a$ to occur independently, which is a requirement for proper representation of transient phenomena (Cornaton, 2012). Overwhelmingly, RTDs have been assumed to be at steady-state. This removes the temporal derivative from (1) and $\rho_{w}$ no longer includes time, so equation (1) mimics the classical 4-D continuity equation, with residence time substituting for time:

$$
\frac{\partial \rho_{w}}{\partial a}+\nabla \cdot \mathbf{j}_{w}\left(\rho_{w}\right)=0
$$

The steady-state assumption provides a significant advantage because RTDs become analogous to Green's functions of transport equations for the water mass itself. Furthermore, solutions of 4-D transport problems are much simpler than 5-D problems, many of which may even have analytical solutions. The preponderance of the steady-state assumptions in the literature, and the tractable nature of the associated solution techniques, is the motivation for choosing to restrict our discussion to steady-state RTDs in this article, which presents solutions of (2) for problem specific conditions.

\subsection{Eulerian framework}

The Eulerian approach fixes the spatial coordinates of the system and considers how mass moves within a given volume and exchange between "external" elements (i.e. sources, sinks, boundaries, etc...). The fundamental equation for the steady-state RTD in Steady-state analytical RTDs 
the Eulerian reference frame, known as "age equation", can be written as (Ginn, 1999; Ginn et al., 2009):

$$
\frac{\partial \rho_{w}(\mathbf{X}, a)}{\partial a}=-\nabla \cdot \mathbf{j}_{w}\left(\rho_{w}(\mathbf{X}, a)\right)
$$

with boundary conditions:

$$
\begin{gathered}
\rho_{w}(\mathbf{X}, a)=\delta(a) \quad \mathbf{X} \in \boldsymbol{\Gamma}_{\boldsymbol{r}} \\
\rho_{w}(\mathbf{X}, a)=\rho_{b}(\mathbf{X}, a) \quad \mathbf{X} \in \boldsymbol{\Gamma}_{\boldsymbol{b}}
\end{gathered}
$$

where $\delta$ is the Dirac delta distribution and $\mathbf{X}=\left(x_{1}, x_{2}, x_{3}\right)$ is the spatial location. The transport operator on the right hand side $\nabla \cdot \mathbf{j}_{w}[*]$ represents any transport mechanism, which may consist of advection and Fickian dispersion or other forms of transport such as anomalous dispersion. $\boldsymbol{\Gamma}_{\boldsymbol{r}}$ is the surface recharge boundary and $\boldsymbol{\Gamma}_{\boldsymbol{b}}$ represent the internal boundaries where the RTD of inflowing water is known. Other forms of boundary condition including flux or symmetrical boundary conditions can also be considered which are not presented for the sake of brevity. As said above, replacing $a$ with $t$ in Eq. (3) makes it identical to the equation for transport of a conservative, non-reactive tracer with a Dirac spike boundary condition at the recharge boundary. This provides a simple practical way to model steady-state RTDs in complex systems using already available contaminant transport codes. However representing a Dirac delta distribution as the boundary condition may pose some numerical challenges and it may be easier to model the cumulative residence time instead. Integrating Eq. (3) for the sake of generality with respect to $a$ yields:

$$
\frac{\partial \mathrm{P}_{w}(\mathbf{X}, a)}{\partial a}=-\nabla \cdot \mathbf{j}_{w}\left(\mathrm{P}_{w}(\mathbf{X}, a)\right)
$$

with a Heaviside recharge boundary:

$$
\mathrm{P}_{w}(\mathbf{X}, a)=H_{s}(a) \quad \mathbf{X} \in \Gamma_{r}
$$




$$
\mathrm{P}_{w}(\mathbf{X}, \mathrm{a})=\mathrm{P}_{b}(\mathbf{X}, \mathrm{a}) \quad \mathbf{X} \in \boldsymbol{\Gamma}_{\boldsymbol{b}}
$$

where $\mathrm{P}_{w}(\mathbf{X}, a)=\int_{0}^{a} \rho_{\mathrm{w}}(\mathbf{X}, a) d a$ is the cumulative RTD and $H_{s}$ is the Heaviside or step function.

\subsection{Lagrangian approach}

The Lagrangian approach to residence time can be thought of as a discretization of the mass of the system whereas the Eulerian approach is a discretization of space; note that the term "discretization" is used loosely here since all of the equations are valid for finite and infinitesimal (continuum) masses and volumes, respectively. The governing equations for both of these reference frames are similar and both require similar information about the fluxes of water. The subtle difference is that a discrete volume of water is tagged and followed through the hydrologic system at the mass-averaged (barycentric) velocity (Ginn, 1999), instead of moving that water through a fixed volume (Eulerian). In reality, the water within the tagged volume is not isolated and experiences diffusive exchange with the neighboring volumes it encounters, so the distribution of residence time within a Lagrangian element is not strictly constant over time. If one assumes that the diffusive exchange of adjacent volumes is similar in magnitude and that their RTDs are only slightly different, the diffusive fluxes between the neighboring volumes can be assumed to be in a local dynamic equilibrium, meaning variables pertaining to the problem are continuous. This is similar to the so-called "paradox of groundwater age" described by Bethke and Johnson (2002). However, the diffusive fluxes are less likely to balance at larger scales or over long distances and significant variations of the RTDs over short distances may be observed (Varni and Carrera, 1998). This leads to the question of volume averaging, which is briefly discussed later.

One of the simplest approaches for deriving a Lagrangian equation for residence time is the discrete random walk, demonstrated by Engdahl et al. (2012) for the fully transient case. Readers are referred to that article for the details of the derivation, as well as spatially and temporally continuous random walk derivations, but the basic approach assumes that the fluid velocity is deterministic and that diffusive/dispersive fluxes are probabilistic. This means solving a diffusion equation in the local Lagrangian coordinates 
instead of an advection-dispersion equation. A complimentary approach is based on the conditional probability that a particle occupying some discrete spatial location, $\mathbf{X}=$ $\left(x_{1}, x_{2}, x_{3}\right)$, came from another nearby location one time step earlier. If the system is steady-state with respect to residence time, this probability is:

$$
\rho_{p a}(\mathbf{X}, a+\Delta a)=\sum_{\Delta \mathbf{x}} p(\Delta \mathbf{x}) \rho_{p a}(\mathbf{X}-\Delta \mathbf{X}, a)
$$

where $p(\Delta \mathbf{x})$ is the probability mass function of the possible displacements, $\Delta \mathbf{x}$ is the jump length vector, $\rho_{p a}$ denotes probability of a particle, and $a$ is the variable for residence time. This is an accounting of all the possible, previous spatial locations in $d$ dimensions and the form of $p(\Delta \mathbf{x})$ dictates whether these probabilities will be asymmetric or anisotropic. The governing equation can be generated from a first-order Taylor expansion of the probability terms in residence time:

$$
\rho_{\mathrm{pa}}(\mathbf{X}, a+\Delta a) \approx \rho_{\mathrm{pa}}(\mathbf{X}, a)+\Delta a \frac{\partial \rho_{\mathrm{pa}}}{\partial a}
$$

and a second-order expansion in space:

$$
\rho_{\mathrm{pa}}(\mathbf{X}-\Delta \mathbf{x}, a) \approx \rho_{\mathrm{pa}}(\mathbf{X}, a)-\Delta \mathbf{x} \cdot\left(\nabla \rho_{\mathrm{pa}}\right)+\frac{\Delta \mathbf{x} \Delta \mathbf{x}^{\mathbf{T}}}{2}:\left(\nabla \nabla^{\mathrm{T}} \rho_{\mathrm{pa}}\right)
$$

where ${ }^{\mathrm{T}}$ denotes a vector transpose. These approximations are substituted into Eq. (9) to produce a form of Eq. (2):

$$
\frac{\partial \rho_{p a}}{\partial a}+\mathbf{v}_{\mathbf{x}} \cdot\left(\nabla \rho_{p a}\right)-\mathbf{D}_{\mathbf{x}}:\left(\nabla \nabla^{\mathrm{T}} \rho_{p a}\right)=0
$$

where $\mathbf{v}_{\mathbf{x}}=\lim _{\Delta \mathbf{x} \rightarrow \mathbf{0}}\langle\Delta \mathbf{x}\rangle / \Delta t, \quad \mathbf{D}_{\mathbf{x}}=\lim _{\Delta \mathbf{x} \rightarrow \mathbf{0}}\left\langle\Delta \mathbf{x} \Delta \mathbf{x}^{\mathbf{T}}\right\rangle / 2 \Delta t, \quad$ angle brackets denote expected values and a continuum limit is invoked. Strictly $\rho_{p a}$ is the probability of finding a single particle at location $\mathbf{X}$ with residence time $a$, so an ensemble average over a large number of independent, identically distributed particles gives the residence time distribution.

Steady-state analytical RTDs 
The most common use of the Lagrangian reference frame is for tagged particle approaches to generate RTDs at target points or a domain wide flux distribution. Applications of particle tracking in the context of residence time are plentiful (Engdahl and Maxwell, 2015; Kollet and Maxwell, 2008; Tompson et al., 1999; Weissmann et al., 2002) but these are merely numerical solutions of the governing equations along the characteristics. Physically, these characteristics are streamlines and it is often possible to solve the Lagrangian equations analytically along streamlines (see section 3.2).

\subsection{Averages of RTDs}

RTDs reported in the literature are predominately some kind of volume average, and this broad definition includes averages over the water exiting a domain. Since the governing equations describe a spatially distributed function, it is worth considering the conditions under which different volume averages are, or may become equivalent.

Volume averaged RTDs should represent the distribution of all fluid residence times within the volume; this is in contrast to the mean of an RTD which is a scalar. Assume that the RTD at every point within a 3-D hydrologic system is a solution of equation (3), and that they are defined everywhere in the system. The RTD of any subdomain within the system includes all the RTDs within the subdomain, and is found as a weighted average (e.g. Quintard and Whitaker, 1993). This average can also be found by taking the expected value of the "age equation" itself. If all of the individual RTDs are known exactly, there will be no error in the volume averaged RTD, regardless of the size of averaging volume. The mean of the averaged distribution will be the weighted average of the individual means, with similar equivalences for higher moments.

The main issue with volume averages is how to compare one to another and this has caused much confusion in the literature. For example, given some system, a domain wide approach such as in Botter et al. (2011) is equivalent to an average over a solution of equation (2) such as in Benettin et al. (2013), so it is possible to compare RTDs at the system's outlet or at a sampling device to spatial averages (Etcheverry and Perrochet, 2000). However, this equivalence can be difficult to show formally for specific RTDs as it requires a complete knowledge of the system and all its boundaries as well as closed- 
form expressions for system's characteristics and resulting RTDs, which is practically impossible. Whenever a volume averaged RTD is reported or defined, the conditions of that average should be carefully considered. It is also important to consider whether any summary statistics (e.g. mean and variance) are representative of the RTD or if the full distribution is needed. For example, the mean residence time of a bimodal distribution could have a density of zero, meaning that none of the water is actually associated with that value. The mean residence time can also be strongly impacted by the tail of the distribution (Bethke and Johnson, 2002), which is often difficult to characterize. These issues may be particularly important in integrated hydrologic systems with broad RTDs (e.g. Engdahl and Maxwell, 2014; Kollet and Maxwell, 2008). When comparing averaged distributions, it is worthwhile to take a reductionist approach and consider how that average would be constructed from a large ensemble of fluid parcels, at least conceptually. Individual parcels are the building block for all RTDs and they provide a unifying framework across disciplines, which should help resolving any confusion about different definitions.

Lastly, it should be reiterated that RTD estimates are not error free and an analytical model is often assumed (Section 3). Any error in the parameters of the analytical model, the form of the analytical function, or even the number of analytical models (Section 4) will affect the average and these errors will propagate. This is another example where the reductionist approach is particularly useful.

\section{Steady-state RTDs and the natural world: basic analytical solutions}

This section presents steady-state solutions for residence time distribution. Well-known solutions are introduced as well as a few little known - still useful - ones. For each solution, the framework for their applicability is provided along with the mathematical formula for the RTD and the mean residence time. We specifically highlight the effect of the controlling parameters on the shape of the RTD and compare RTDs with each other. Except for the system presented in section 3.2.2 which gives the well-known exponential model, the derivation of the RTDs is not provided; it can be either found in the literature or easily derived from the exponential model. This section is largely "ready-to-use" for those wishing to pick an RTD model for an application.

Steady-state analytical RTDs

Page 12 of 77 


\subsection{Globally-mixed systems}

A perfectly mixed system is defined as a system for which any fluctuation in the input concentration of a compound is instantaneously averaged across the whole system as the result of internal mixing mechanisms (Danckwerts, 1953). Purposely-mixed vessels such as batch reactors or blenders found in chemical engineering are examples of systems that can be idealized as perfectly-mixed systems or more generally globally-mixed systems, as mixing might not be perfect in practice. Following works of Danckwerts (1953) and von Buttlar and Libby (1955), Wolf and Reisnick (1963) proposed a general formula for residence time distribution at the outlet of globally-mixed systems $\rho_{T}(a)\left[\mathrm{T}^{-1}\right]$. The formula stems directly from experimental data on batch reactors for which an affine transformation between $\ln \left(1-\mathrm{P}_{T}(a)\right)$ and $\frac{a}{\beta}$ has been observed when applying a solute impulse at the inlet, where $\mathrm{P}_{T}(a)$ is the cumulative residence time distribution at the outlet and $\beta$ is the volume of the system divided by the flow rate. The steady-state RTD at the outlet of a globally-mixed system then takes the form of an exponential function (Figure 2):

$$
\left\{\begin{array}{c}
\rho_{T}(a)=\frac{\eta}{\beta} \exp \left(-\eta \frac{a-\varepsilon}{\beta}\right), a \geq \varepsilon \\
\rho_{T}(a)=0, a<\varepsilon
\end{array}\right.
$$

where $\beta[\mathrm{T}], \eta[-]$ and $\varepsilon[\mathrm{T}]$ are the three controlling parameters. The mean residence time $\tau[\mathrm{T}]$ is defined by:

$$
\tau=\int_{\varepsilon}^{+\infty} a \rho_{T}(a) d a=\frac{\beta}{\eta}+\varepsilon
$$

Eq. (9) has not been derived analytically but Wolf and Reisnick (1963) provided meaningful physical interpretations for $\eta$ and $\varepsilon . \eta$ can be viewed as the measure of the mixing efficiency within the system: a perfect mixing would correspond to $\eta$ equal to 1 while $\eta$ tending to infinity would imply no mixing at all. $\varepsilon$ can be viewed as the measure of the system phase shift, either a delay (positive value) or an anticipation (negative value). The generality of the two parameters $\eta$ and $\varepsilon$ allows taking into consideration Steady-state analytical RTDs 
various phenomena leading to a deviation from the perfect-mixing situation (in which $\eta=1, \varepsilon=0$ ) such as dead space, short circuiting, additional plug flow or system lag (Figure 2). Eq. (9) can be obtained with the mass balance of Eq. (2) lumped over the entire reactor and applying $\nabla \cdot \mathbf{j}_{w}\left(\rho_{w}\right)=\frac{\rho_{w}}{\beta}$, the latter term corresponding to the loss of water mass at the outlet.

By extension, it has often been suggested that the exit RTD of hydrologic systems could be compared to an exponential model and that the degree of resemblance would be indicative of the degree of mixing occurring in the system (Amin and Campana, 1996; Begemann and Libby, 1957; Danckwerts, 1953). However, great care has to be taken when doing such interpretation as other types of systems (i.e. not globally-mixed) can also feature an exponential exit RTD. This is the case of aquifers subject to uniform recharge and under the Dupuit assumption (see below), in which case an exponential behavior emerges from the particularities of the advection process and discharge conditions and not from mixing within the system (Eriksson, 1958; Haitjema, 1995). More generally, hydrologic systems are often characterized by a dominance of advection over mixing at the scale of the system, meaning that mixing occurs only locally at relatively small scales. In this case, the exit RTD should not be interpreted in terms of (global) mixing efficiency. By way of proof, the systems presented in the following section are all often qualified as "full-mixing" in the sense the RTD is obtained gathering all flow lines, and though their RTD can strongly differ from an exponentially decreasing function only because of the system's properties. The full-sampling of flow lines only guarantees that the RTD will indeed be a distribution: indeed, the more flow lines are sampled, the broader the RTD is expected to be. But it strongly comes back to the boundary conditions and the system's properties to affect the shape of the RTD.

\subsection{Purely-advective systems}

The compliment to perfect-mixing is no mixing where all molecules/particles of the system are assumed to move at the local velocity of the bulk fluid. The lack of mixing implies that no dispersion or diffusion is occurring, so these systems are said to be purely-advective. The corresponding form of Eq. (2) is achieved with the 
definition $\mathbf{j}_{w}\left(\rho_{w}\right)=v(\boldsymbol{x}) \rho_{w}$, where $v(\boldsymbol{x})\left[\mathrm{LT}^{-1}\right]$ is the fluid velocity vector field. In reality, all systems are dispersive to some degree because: (i) the individual molecules/particles constituting any volume are animated by Brownian motion implying that they can divert from the bulk direction of the fluid (i.e. diffusion occurs); and (ii) hydrological systems are generally described at a scale at which the velocity is understood in an average sense (following the classical concept of a representative elementary volume (Bear, 1972)), implying that small-scale heterogeneities of the velocity field induce mixing at the scale of description (i.e. mechanical dispersion occurs). Despite these points, the conceptual model of purely-advective systems is useful at least because they provide a reasonable approximation for systems with minimal dispersion (i.e. with high Peclet number).

\subsubsection{Stream tubes}

The lack of internal mixing in purely-advective systems reduces the RTD of an infinitesimal volume at any non-singular point of the system to the Dirac delta function $\delta$. The unique residence time $a$ is a simple function of the velocity along the infinitesimal stream tube:

$$
a\left(s_{i}, s_{o}\right)=\int_{s_{i}}^{s_{o}} \frac{1}{v(s)} d s
$$

where $s[\mathrm{~L}]$ is an integration variable representing the distance along the stream tube from the entry point $s_{i}[\mathrm{~L}]$ to the point of interest $s_{o}[\mathrm{~L}], v(s)\left[\mathrm{L} \mathrm{T}^{-1}\right]$ is the velocity along the stream tube. When taken at the outlet, the RTD hence reverts to the Dirac delta function $\delta$ (Figure 3a) provided the stream tube is effectively the only one sampled and that there is no internal source or sink. In the trivial case where the velocity is constant along a flow line - i.e. if the cross-sectional area of an infinitesimal stream tube is constant - the unique residence time, which is besides the mean residence time, is simply given by:

$$
\tau=\frac{\theta L}{R}
$$

Steady-state analytical RTDs

Page 15 of 77 
where $\theta[-]$ is the effective porosity for porous media ( $\theta=1$ otherwise), $L[\mathrm{~L}]$ is the length of the stream tube, and $R\left[\mathrm{~L} \mathrm{~T}^{-1}\right]$ is the input flux (for instance, the recharge to a semi-confined or unconfined aquifer). If the cross-sectional area is decreasing or increasing linearly along the stream tube - from $A_{o}$ to $A_{i}\left[\mathrm{~L}^{2}\right]$, and assuming a constant effective porosity, the unique (mean) residence time is given by (Figure 3a):

$$
\tau=\frac{\theta L}{2 R}\left(1+\frac{A_{o}}{A_{i}}\right)
$$

Such systems are often referred to as piston flow (Danckwerts, 1953; Raats, 1977b). However, the sampling conditions of natural systems are in practice likely to gather various flow lines coming from different origins, and so equations (12) and (13) should be used with great care. It is only applicable for a few cases, typically for homogeneous confined aquifers with possibly varying thickness (Etcheverry, 2001), aquitards where mass transfer with the surroundings compartments can be neglected (Bethke and Johnson, 2002; Castro et al., 1998), highly localized samplings close to the system inlet (Leray et al., 2012; Marçais et al., 2015), or fractured and karst systems (Bockgård et al., 2004; Burton et al., 2002; Knowles et al., 2010; Long and Putnam, 2006; Long and Putnam, 2009).

Equation (11) highlights the fact that the RTD for an ensemble of stream tubes of a purely-advective system can be found if the flow lines are known together with the velocity along them. On this point, Raats in a series of papers $(1974 ; 1977 \mathrm{a} ; 1977 \mathrm{~b})$ expressed the residence time as a function of the cross sectional area of the stream tube $A(s)$ resulting in:

$$
a\left(s_{i}, s_{o}\right)=\left(\frac{1}{\theta v A}\right)_{i} \int_{s_{i}}^{s_{o}} \theta A(s) d s
$$

where the lower script $i$ denotes a variable taken at the start of the stream tube. Equation (14) is similar to equation (11) considering mass conservation of water along the stream tube. Raats further showed that the stream tube area can be related to the unit tangent 
velocity field along the stream tube $\boldsymbol{u}$ - which is simply the velocity field divided by its magnitude:

$$
A\left(s_{i}, s_{o}\right)=A_{i} \exp \left[\int_{s_{i}}^{s_{o}} \nabla \cdot \boldsymbol{u} d s\right]
$$

$\nabla \cdot \boldsymbol{u}$ is in fact the measure of the divergence $(\nabla \cdot \boldsymbol{u}>0)$ or the convergence $(\nabla \cdot \boldsymbol{u}<0)$ of the stream tube. Variations of the stream tube area $A\left(s_{i}, s_{o}\right)$ occur due to changes in the system's geometry and boundary conditions along with flow rate of all adjacent stream tubes. In systems where the boundary conditions and properties are described by simple functions, analytical forms of the geometry of each stream tube can be found, which then provides the residence time at any point. When considered at the system's outlet or at any non-local sampling device which is very often the case in hydrologic systems, the RTD will be a flux-averaged ensemble of all the stream tubes reaching the sampling zone. Under steady-state conditions, the cumulative RTD $\mathrm{P}_{T}(a)$ can then be expressed as the outflow rate having a residence time inferior or equal to $a, Q_{o u t}(a)$, divided by the total outflow rate $Q_{\text {out }}\left[\mathrm{L}^{3} \mathrm{~T}^{-1}\right]$ (Etcheverry and Perrochet, 2000):

$$
\mathrm{P}_{T}(a)=\frac{Q_{\text {out }}(a)}{Q_{\text {out }}}
$$

The residence time distribution $\rho_{T}(a)$ is the derivative of $\mathrm{P}_{T}(a)$ with respect to $a$ :

$$
\rho_{T}(a)=\frac{d \mathrm{P}_{T}(a)}{d a}=\frac{1}{Q_{\text {out }}} \frac{d Q_{\text {out }}(a)}{d a}
$$

Therefore, if an analytical solution can be found for $Q_{o u t}(a)$, the RTD at the outlet of the system can be derived analytically. This is possible for a number of idealized hydrologic systems, as shown hereafter.

\subsubsection{Aquifers under uniform recharge conditions and constant properties}

Several authors showed that the RTD can take the form of an exponential function (Figure $3 \mathrm{~b}$ ) if the following conditions/assumptions are satisfied: 1) there is local 
homogeneity (i.e. no stratification), 2) the Dupuit assumption holds (i.e. constant hydraulic gradient along the vertical), 3) the recharge rate $R$ and $\frac{\theta H}{R}$ are constant with $H$ the saturated thickness and $\theta$ the effective porosity, 4) flow lines are sampled over the entire saturated thickness - this implies that the RTD may be obtained at the system outlet or at a fully-penetrating well (Haitjema, 1995; Leray et al., 2012; Raats, 1977b; Vogel, 1967).

\subsubsection{Derivation and properties}

We derive here the flux-averaged RTD in one dimension and Cartesian coordinates as this subsequently allows some of the assumptions to be relaxed (see sections 3.2.3, 3.2.4 and 4.3). The reader is referred to Haitjema (1995) for a demonstration in two dimensions (i.e. in the horizontal plane). Consider then a homogeneous 1-D aquifer of constant saturated thickness $H$ [L] (Figure 4) with no internal source or sink. Given a constant recharge rate $R\left[\mathrm{~L} \mathrm{~T}^{-1}\right.$ ), the total inflow is a linear function of the position $x$ from the farthest recharge point:

$$
Q_{\text {in }}(x)=R x
$$

There is no variation of the storage under steady-state conditions and so the total outflow rate equals the total inflow rate. A fully-penetrating well - pumping or not - located at position $x=L$ and catching all flow lines then sees a quantity $Q_{\text {in }}(L)=Q_{\text {out }}(L)=R L$ of water. Under the Dupuit assumption, the horizontal velocity $v(x)$ is constant with depth and therefore:

$$
v(x)=\frac{R x}{\theta H}
$$

It follows that every parcel of water infiltrating at the position $x$ and traveling with advection only takes the following time to reach the observation position $L$ when $\frac{\theta H}{R}$ is constant throughout the domain:

Steady-state analytical RTDs

Page 18 of 77 


$$
a(x)=\int_{x}^{L}\left(\frac{R x}{\theta H}\right)^{-1} d x=\frac{\theta H}{R} \ln \frac{L}{x}
$$

Water infiltrating at $x=L$ has a null residence time, while water infiltrating at $x=0$ has an infinite residence time. Equivalently, the infiltration position $x(a)$ of water exiting the system with a residence time $a$ is given by:

$$
x(a)=L \exp \left[-\left(\frac{\theta H}{R}\right)^{-1} a\right]
$$

The outflow having a residence time inferior or equal to $a$ is:

$$
Q_{\text {out }}(a)=R L-R x(a)
$$

Therefore, the outlet RTD $\rho_{T}(a)$ (Figure 3b) can be expressed using equation (17) and with the condition of a constant recharge rate $R$ as:

$$
\rho_{T}(a)=\frac{1}{R L} \frac{d(R L-R x)}{d a}=-\frac{1}{L} \frac{d x}{d a}=\frac{1}{\tau} \exp \left(-\frac{a}{\tau}\right)
$$

where $\tau$ is the mean residence time which directly relates to system's properties and boundary conditions:

$$
\tau=\frac{\theta H}{R}
$$

Besides, it can be verified that when multiplying the numerator and the denominator by the contributing area, equation (24) gives $\tau=V / Q_{\text {out }}$, with $V$ the volume of the aquifer.

Haitjema (1995) showed that this result is valid for 2-D horizontal aquifers of any size and any shape. Indeed, while the size $L$ of the system is used here to derive the RTD (see equation (20)), it finally vanishes in the formulas of the RTD and of the mean residence time. Besides, the result holds whatever the type of outlet: for the discharge to a stream network as well as for the flow to a well (pumping or not). In all rigors, the result is valid only for fully-penetrating features (i.e. features that are connected to the aquifer over its Steady-state analytical RTDs 
entire thickness). Nevertheless, Luther and Haitjema (1998) suggested that the error in the case of partially-penetrating or surface discharge features such as stream network is negligible when the outlet area is less than $10 \%$ of the system area, as long as the discharge features still capture all the flow lines. The main criteria for the exponential form to hold are that the recharge rate $R$ and the ratio $\frac{\theta H}{R}$ remain constant throughout the system to get closed-form expressions for the residence time (Eq. (20)) and the RTD (Eq. (23)) respectively. Non-compliance of the two criteria entails the deviation of the RTD from the exponential form given by Eq. (23) as shown in sections 3.2.3 and 3.2.4.

\subsubsection{The case of partially-penetrating observation wells}

An observation well (i.e. a non-pumping well) from which a sample is taken can be seen as an ephemeral discharge feature which captures at least part of the flow lines, at most, all flow lines without disturbing the flow pattern. In the latter case, as said above, the RTD of the sample also takes the form of an exponential function. But in the case of a partially-penetrating observation well, only the flow lines that cross the well screen are captured and not the ones above or below; and this affects the RTD.

In the case where the well is screened over the bottom part of the aquifer, the time required for groundwater to reach the top of the screen results in a global delay $a_{u p}[\mathrm{~T}]$ that needs to be taken into account. The shift $a_{u p}$ corresponds to the shortest residence time detected by the observation well and is associated to the shortest flow path screened coming from the nearest recharge point. It depends on the system properties (thickness, effective porosity) as well as on boundary conditions (recharge rate). In the case of a 1-D aquifer such as above, $a_{u p}$ is given by:

$$
a_{u p}=\frac{\theta H}{R} \ln \frac{1}{1-C_{N S}}
$$

where $C_{N S}[-]$ is the portion of saturated thickness that is not sampled; it is here $\frac{H_{u p}}{H}$ (Figure 4). The RTD appears to be lagged (Figure 3b): 


$$
\left\{\begin{array}{c}
\rho_{T}(a)=\left(\frac{\theta H}{R}\right)^{-1} \quad \exp \left[-\left(\frac{\theta H}{R}\right)^{-1}\left(a-a_{u p}\right)\right], a \geq a_{u p} \\
\rho_{T}(a)=0, a<a_{u p}
\end{array}\right.
$$

The mean residence time $\tau$ is given by:

$$
\tau=\frac{\theta H}{R}+a_{u p}
$$

When $C_{N S}$ tends to 0 , so does $a_{u p}$ and $\tau$ tends to $\frac{\theta H}{R}$ as for a fully-penetrating well (equation (24)).

In the case where the well is screened over the top part of the aquifer (Figure 4), the deepest flow lines with a residence time higher than $a_{\text {low }}$ are not sampled by the well:

$$
a_{\text {low }}=-\frac{\theta H}{R} \ln C_{N S}
$$

where $C_{N S}$ is here $\frac{H_{\text {low }}}{H}$ (Figure 4$)$. The RTD has to be corrected of these non-sampled flow lines and hence it appears rescaled and truncated (Figure 3b):

$$
\left\{\begin{array}{c}
\rho_{T}(a)=\frac{1}{1-C_{N S}}\left(\frac{\theta H}{R}\right)^{-1} \exp \left[-\left(\frac{\theta H}{R}\right)^{-1} a\right], a \leq a_{l o w} \\
\rho_{T}(a)=0, a>a_{l o w}
\end{array}\right.
$$

The mean residence time $\tau$ is given by:

$$
\tau=\frac{\theta H}{R}-a_{\text {low }} \frac{C_{N S}}{1-C_{N S}}
$$

When $C_{N S}$ tends to 0 , so does $a_{\text {low }} C_{N S}$ and $\tau$ tends to $\frac{\theta H}{R}$ as for a fully-penetrating well.

These last two examples are indicative of the importance of taking the sampling conditions under careful consideration as their effect on RTD is clear: depending on the well casing, the RTD is shifted towards larger residence times or rescaled and truncated 
to shorter residence times (Figure 3b); the same applies to the mean residence time as showed by equations (28) and (30).

\subsubsection{Aquifers under linearly-variable recharge conditions and constant properties}

Etcheverry (2001) considered the case of 1-D horizontal aquifers recharged at a linearlyvariable rate, either increasing or decreasing towards the discharge zone (Figure 3c). The assumptions to derive the RTD at the discharge zone are the same as above with the exception of the non-uniform input flux $R(x)$ which is expressed by:

$$
R(x)=R_{0}+\frac{x}{L}\left(R_{L}-R_{0}\right)
$$

where $x$ is the longitudinal position, $L$ is the longitudinal extension of the system, $R_{L}$ and $R_{0}$ are the recharge rates $\left[\mathrm{L} \mathrm{T}^{-1}\right]$ at the downstream and upstream boundaries, respectively. The RTD at the outlet catching all flow lines is then given by:

$$
\rho_{T}(a)=\frac{4 R_{0}^{3}}{\theta H} \exp \left(\frac{R_{0}}{\theta H} a\right) \frac{\left(R_{L}+R_{0}\right) \exp \left(\frac{R_{0}}{\theta H} a\right)+\left(R_{L}-R_{0}\right)}{\left(\left(R_{L}+R_{0}\right) \exp \left(\frac{R_{0}}{\theta H} a\right)-\left(R_{L}-R_{0}\right)\right)^{3}}
$$

and the mean residence time $\tau$ is a simple function of the mean recharge rate, the effective porosity and the thickness:

$$
\tau=\frac{\theta H}{\frac{R_{L}+R_{0}}{2}}
$$

As for aquifers under uniform recharge conditions, the formulas for the RTD under linearly-variable recharge conditions and for the mean residence time are both independent of the size of the aquifer $L$. The non-uniform recharge rate distorts the RTD around the solution obtained from the uniform-recharge case which is retrieved from Equation (32) when $R_{L}=R_{0}$ (Figure 3c). For systems where the recharge rate increases towards the discharge zone $\left(R_{L}>R_{0}\right.$ ), water entering the system near the discharge zone flows faster than in the uniform case resulting in a higher portion of young water Figure 
$3 c)$. Inversely, for systems where the recharge rate decreases towards the discharge zone ( $R_{L}<R_{0}$ ), water entering the system far from the discharge zone flows faster than in the uniform case, so that the proportion of old water is higher (Figure 3c).

\subsubsection{Trapezoidal and wedge aquifers under uniform recharge conditions}

Etcheverry (2001) and later Leray et al (2012) developed a model for 1-D horizontal systems in which the thickness $H(x)$ is linearly variable, either increasing or decreasing towards the discharge zone; in other words, for trapezoidal systems (Figure 3d):

$$
H(x)=H_{0}+\frac{x}{L}\left(H_{L}-H_{0}\right)
$$

where $H_{L}$ and $H_{0}$ are the system thicknesses [L] at its downstream and upstream boundaries, respectively. Provided the recharge rate and the system's properties remain uniform, the RTD at the outlet catching all flow lines is:

$$
\rho_{T}(a)=\frac{R}{\theta\left(H_{L}-H_{0}\right)} \frac{f(a)}{1+f(a)}
$$

where $f(a)$ is the unique real positive solution of the following equation, also known as the Lambert function:

$$
f(a) \exp (f(a))=\frac{H_{L}-H_{0}}{H_{0}} \exp \left(-\frac{R}{\theta H_{0}} a+\frac{H_{L}-H_{0}}{H_{0}}\right)
$$

Here as well, the RTD formula is independent of the size of the aquifer $L$. So is the mean residence time $\tau$ whose expression is:

$$
\tau=\frac{\theta \frac{H_{L}+H_{0}}{2}}{R}
$$

The shape of the residence time distribution strongly evolves from a step function from 0 to $2 \tau$ (when the upstream thickness $H_{0}$ is negligible relatively to the downstream thickness $H_{L}$ ) to an exponentially decreasing function otherwise (Figure 3d). When the thickness decreases towards the discharge zone, the horizontal velocity sharply increases 
close to the discharge zone because of the strong convergence of flow paths, resulting in a high proportion of young water (Figure 3d). This illustrates the concept detailed in section 3.2.1. On the contrary, an increasing thickness implies a relatively lower velocity close to the discharge zone because of the divergence of flow paths, resulting in a lower proportion of young water. The RTD for this case converges toward a step function as the upstream thickness tends to 0 , in which case a wedge-aquifer configuration is retrieved. Eriksson (1958) already developed a solution for this configuration in which the streamlines are parallel to each other, and also parallel to the stratum. The residence time along the vertical at the outlet is then simply a linear function of the distance from the recharge zone (Cook and Böhlke, 2000; IAEA, 2006) and the residence time distribution is given by:

$$
\rho_{T}(a)=\frac{H_{s}(2 \tau-a)}{2 \tau}, a \geq 0
$$

where $H_{S}$ is the Heaviside step function; this is commonly referred to as the "linear" model because of the linear dependence of $a$ to the position $x$. In this case, the mean residence time $\tau$ is simply:

$$
\tau=\frac{\theta H_{L}}{2 R}
$$

where $H_{L}$ is the aquifer thickness at the discharge.

Studies in the literature have traditionally focused on the dependence of the RTD on the recharge rate which is hence often preferentially determined with tracers data (Carrera and Varni, 2000; Newman et al., 2010; Sanford, 2011; Zuber et al., 2011). However, the solution for trapezoidal systems demonstrates the importance of the system's geometry on the spatial evolution of the flow field, specifically on the divergence or convergence of flow paths. The RTDs are indeed the result of the complex interactions between the intensity of the pore velocity and the shape of the flow paths, i.e. the flow pattern. This is of importance for systems characterization as, under certain conditions, one may prefer to constrain the system's geometry rather than recharge conditions (Leray et al., 2012).

Steady-state analytical RTDs

Page 24 of 77 


\subsubsection{General considerations on the systems presented so far}

Conceptually, the results of these sections apply to both unconfined and semi-confined aquifers. However, one must keep in mind that the RTD reflects only the time spent in the system under consideration. For unconfined aquifers, the inflow occurs at the water table. The concentration of environmental tracers can be generally known there, provided some "alterations" of the atmospheric input caused by changes in recharge temperature, sorption on soil materials, excess air amongst others (IAEA, 2006) are characterized or negligible (Suckow, 2014). Under those conditions, RTD models of unconfined aquifers can be used to interpret environmental tracer data (Małoszewski and Zuber, 1982). In contrast, for semi-confined aquifers, the inflow occurs via leakage from an overlying aquifer or from adjacent systems so that the input concentration of environmental tracers needs to be specifically assessed at the confined system boundaries. Therefore, the use of RTD models of semi-confined aquifers may encounter practical limitations. Also note that accordingly, groundwater residence time is classically defined as the time spent by the water in the subsurface after it reaches the water table (Suckow, 2014), i.e. the concept of groundwater residence time implies the consideration of unconfined conditions.

\subsubsection{Confined aquifer with a pumping well}

A simple but informative case is the one of radial flow towards a pumping well in a confined aquifer (Figure 3e). If the effective thickness $(\theta H)$ is constant, the velocity is simply given as:

$$
v(r)=\frac{Q_{\text {out }}}{2 \pi r \theta H}
$$

where $Q_{o u t}$ is here the pumping rate, and is $r[\mathrm{~L}]$ the radial distance from the well. As all flow lines have the same velocity and the same length, there is a unique travel time. The RTD at the pumping well is therefore a Dirac delta distribution where the unique (mean) residence time results from equation (11): 


$$
\tau=\frac{2 \pi \theta H}{Q_{\text {out }}}\left(r_{2}^{2}-r_{1}^{2}\right)
$$

where $r_{1}$ and $r_{2}$ are the well radius and the external radius of the system, respectively. $r_{1}$ is expected to be negligible in practice, but it is kept in the equation for the sake of generality.

\subsubsection{Confined aquifer with a dipole flow field}

Analytical RTDs have been specifically determined for tracer tests interpretation in a dipole flow field (Luo and Kitanidis, 2004). Also referred to as well doublet, a dipole flow field is an extraction-injection well pair. The forced gradient induced by the doublet creates a local recirculation zone whose shape depends on the hydraulic properties of the aquifer and on the regional flow strength and orientation. The tracer tests performed in these conditions are of interest for management of sea water intrusion (Sheahan, 1977), groundwater remediation (Ponsin et al., 2014), reactivity characterization (Burbery et al., 2013) or characterization of hydraulic and transport parameters (Clement et al., 1997; Grove and Beetem, 1971).

In a homogeneous confined aquifer of uniform thickness, and with negligible regional flow, the RTD at the extraction well of the dipole flow field takes the following form (Luo et al., 2007):

$$
\rho_{T}(a)=\frac{1}{\pi} \frac{\sin ^{4}\left[\pi \mathrm{P}_{T}(a)\right]}{\pi \mathrm{P}_{T}(a)-3 \sin \left[\pi \mathrm{P}_{T}(a)\right] \cos \left[\pi \mathrm{P}_{T}(a)\right]+2 \pi \mathrm{P}_{T}(a) \cos ^{2}\left[\pi \mathrm{P}_{T}(a)\right]}
$$

where $\mathrm{P}_{T}(a)$, the cumulative RTD, is solution of the equation:

$$
a=a_{c} \frac{1-\pi \mathrm{P}_{T} \cot \left[\pi \mathrm{P}_{T}\right]}{\sin ^{2}\left[\pi \mathrm{P}_{T}\right]}
$$

where $a_{c}$ is the (median) characteristic time:

$$
a_{c}=\frac{\pi \theta H d^{2}}{Q_{\text {out }}}
$$

Steady-state analytical RTDs

Page 26 of 77 
where $d$ is the distance [L] between the two wells, $H$ is the confined aquifer thickness and $Q_{\text {out }}$ is the extraction flow rate, also equal to the injection flow rate. The system is characterized by a first arrival time equal to a third of $a_{c}$ (Figure $3 \mathrm{f}$ ). It corresponds to the residence time of the shortest flow path connecting the two wells. At late time, the RTD tends to a power-law of slope $-\frac{4}{3}$ (Figure $3 \mathrm{f}$ ) which means that a mean residence time cannot be defined in that case.

Only semi-analytical solutions exist for non-ideal cases, i.e. under regional flow conditions. The reader interested in these solutions are referred to Luo and Kitanidis (2004). Still, as natural systems undergo regional flow, it has to be mentioned that it can significantly affect the shape of the RTD. Depending on the orientation (relative to the wells axis) and strength (relative to the extraction flow rate) of the regional flow, it can distort the flow paths and the velocity within the recirculation zone and may also create capture and release zones (Luo et al., 2006). Possible effects are that a critical flow rate has to be maintained to ensure the existence of a recirculation zone if a uniform regional flow is not exactly oriented in the direction of the injection to the extraction well; or that the first arrival time is impacted by the regional flow orientation: for instance, a countercurrent regional flow will increase the first arrival time; or again, that the powerlaw at late time behavior gives way to an exponential decay which occurs earlier for a lower extraction rate, whatever the regional flow orientation (Luo et al., 2007; Luo et al., 2006).

\subsection{Advective-dispersive systems}

All of the analytical solutions presented in the previous section assume purely-advective flow. Nir (1964) developed an analytical expression for the advective-dispersive RTD. Zuber and coauthors (Kreft and Zuber, 1978; Lenda and Zuber, 1970) later extended this work to either flux or resident boundary conditions. The RTD formulas stem directly from the $\mathrm{ADE}$ of a non-reactive tracer impulse along a semi-infinite one-dimensional flow line. The dispersion is then only longitudinal and Fickian.

At this stage, one has to distinguish between flux and resident concentrations as this may have a significant effect on the RTD when dispersion is not negligible. The former 
$C_{F}(x, t)$ is the mass of solute per unit fluid flux while the latter $C_{R}(x, t)$ represents the mass of solute per unit fluid volume (Parker and van Genuchten, 1984). The two concentrations are easily linked considering mass conservation; the cumulative mass downstream of $x$ at time $t$ equals the total mass of solute that passed through $x$ up to time $t$ :

$$
v \int_{0}^{t} C_{F}(x, t) d t=\int_{x}^{+\infty} C_{R}(x, t) d x
$$

where $v$ is the advective (mean) fluid velocity. The ADE can actually be solved with either concentration (Fienen et al., 2006), with a careful definition of the inlet and sampling conditions when applied to the interpretation of tracer tests. It is highly preferable to treat the inlet boundary of porous media macroscopically to enclose discontinuities coming from the porous structure, and so the inlet boundary will always be some kind of flux condition (Małoszewski and Zuber, 1982), either a third-type boundary when solving the ADE with resident concentration or a first-type boundary when solving with the flux concentration; the two being actually equivalent considering equation (45).

Choosing to solve the $\mathrm{ADE}$ with either the resident or flux concentration actually depends on the sampling conditions. When the tracer concentration is measured in the outflow by extracting a water sample, the sampling gathers different concentrations whose contribution is weighted by the flow rate and the sampling is then said to be flux averaged. In that case, the RTD is obtained solving the ADE with the flux concentration (and using a first-type boundary at the inlet in accordance with aforementioned):

$$
\rho_{T}(a)=\sqrt{\frac{P_{e} \tau}{4 \pi a^{3}}} \exp \left[-(a-\tau)^{2} \frac{P_{e}}{4 a \tau}\right]
$$

where $\tau$ is the mean residence time which is simply $\frac{L}{v}$ with $L[\mathrm{~L}]$ the characteristic flow path length; this is commonly referred to as the "dispersion" model. $P_{\mathrm{e}}[-]$ is the Peclet number, classically defined as the ratio of advective rate to dispersive rate: 


$$
P_{e}=\frac{v L}{D_{l}}
$$

where $D_{l}\left[\mathrm{~L}^{2} \mathrm{~T}^{-1}\right]$ is the longitudinal dispersion. As the Peclet number is defined relatively to the entire flow path, the longitudinal dispersion $D_{l}$ refers to some macroscale dispersion. The dependence of $\tau$ and $P_{e}$ to the flow path length $L$ points out the scaledependence of the solution. The two-parameter distribution of equation (46) has the form of the inverse Gaussian distribution, which is a solution of Eq. (2) with the definition $\mathbf{j}_{w}\left(\rho_{w}\right)=v \rho_{w}+D_{l} \nabla \rho_{w}$; this describes Brownian motion with a drift. Eq. (46) is also equivalent to Eq. 7a in Małoszewski and Zuber (1982).

When the tracer concentration is measured in-situ - by electrical conductivity for instance, it usually involves both the mobile and immobile fluid volume in the vicinity of the sampling (Zhang et al., 2006) which is said to be volume averaged. In that case, the RTD is deduced from solving the ADE with the resident concentration (and using a thirdtype boundary at the inlet):

$$
\rho_{T}(a)=\sqrt{\frac{P_{e}}{\pi a \tau}} \exp \left[-(a-\tau)^{2} \frac{P_{e}}{4 a \tau}\right]-\frac{P_{e}}{2 \tau} \exp \left(P_{e}\right) \operatorname{erfc}\left[(\tau+a) \sqrt{\frac{P_{e}}{4 \tau a}}\right]
$$

Eq. (48) is equivalent to Eq. 7b in Małoszewski and Zuber (1982). The form of the advective-dispersive RTD is strongly impacted by the Peclet number value (Figure 5). As $P e$ tends to infinity, dispersion becomes negligible in comparison to advection and the system can be assumed to be purely-advective whatever the sampling conditions. The RTD tends toward a Dirac delta ditribution with a limited variance of the residence times around the mean residence time $\tau$ (Figure 5). The opposite is observed at small Peclet numbers $(<1)$ where the effect of dispersion becomes pronounced; the RTD is much broader, with a more significant tail and is skewed towards lower residence times (Benettin et al., 2013). Consequently, the proportion of very young water (i.e. with a residence time much lower than $\tau$ ) is significantly higher. The distinction between flux and volume sampling is of importance in these conditions (Figure 5): at high dispersivity, the flux concentration would be rather sensitive to the fastest flow paths while the 
resident concentration would be impacted by slower zones. The determination of the Peclet number and of its component can then be greatly altered by misconsideration of the sampling conditions (Zhang et al., 2006).

These solutions can be advantageously used in advection dominated systems with weak heterogeneities. However, as the RTD is one-dimensional, it applies to localized input zones. If an artificial tracer test is conducted, one may compare the apparent Peclet number with literature values (Visser et al., 2013). Still, when used with environmental tracer data, the apparent Peclet number is highly likely to be overestimated due to the integration over the recharge area. It may also be unreliable because of uncertainties on solute input and not easily linked to evidence of large scale heterogeneities. Non-fickian solutions would be preferred when there is indication of strong heterogeneities resulting in both pronounced preferential flow paths and immobile zones (see section 4.4).

\section{Advanced steady-state analytical solutions}

The RTDs introduced in the previous section are mathematically convenient but are only correct for systems that satisfy the requisite assumptions. Numerically-simulated RTDs and experimental RTDs derived from tracer data are often poorly represented by simple models. Many RTDs in the literature have demonstrated fast arrivals, heavy tails, and multi-modal distributions (Cardenas, 2008; Engdahl et al., 2012; Haggerty et al., 2002; Kirchner et al., 2000; Kollet and Maxwell, 2008; Weissmann et al., 2002). Such features cannot be described efficiently by the simple analytical solutions presented in section 3 . In this section, a number of methods are presented that can be used to derive more advanced analytical solutions. Most of these methods consist of combining simpler solutions in different ways. Therefore, they open the perspective to a quasi-infinite panel of solutions, while still being relatively straightforward to use.

\subsection{Parallel combinations}

In some cases, a system might be decomposed into parallel sub-systems that do not interact but all contribute to the same outlet. The integrated flux RTD at the outlet of such a system is simply a linear combination of the RTDs of the different sub-systems (Figure 6a):

Steady-state analytical RTDs 


$$
\rho_{T}(a)=\sum_{n=1}^{N} \lambda_{n} \rho_{n}(a)
$$

where $\rho_{T}$ is the combined RTD, $\rho_{n}$ denotes the individual distributions of $N$ sub-systems and $\lambda_{n}$ are the weights of each, equal to the relative flux proportions. The only requirement is that each $\rho_{n}$ is a valid probability density distribution and that the weights sum to unity. If each $\rho_{n}$ has an analytical expression, then an analytical expression for $\rho_{T}$ can be derived. Systems presented in section 3 - and more generally, any system whose flow paths are analytically described - can be plugged together to generate composite RTDs. Examples of linear combinations include the exponential and dispersion models (Stolp et al., 2010), exponential and shape-free models (Goderniaux et al., 2013), two piston flow models (Eberts et al., 2012), exponential and piston-flow models (Eberts et al., 2012; Solomon et al., 2010), two exponential-piston-flow models (Green et al., 2014), and multiple dispersion models (Engdahl and Maxwell, 2014; Long and Putnam, 2009; McCallum et al., 2014).

Linear combinations are exceedingly simple yet it has been demonstrated that very complex RTDs can be approximated quite accurately from a linear combination (Engdahl and Maxwell, 2014). In fact, any purely-advective solution (such as the ones presented in section 3) can be constructed as an infinite combination of Dirac delta distributions. Envision a histogram with a finite number of bins and the height of each bar is the number of the flow paths within that bin of residence time. In the limit where the size of each bin approaches zero, the normalized heights of the bins become the density of each residence time. An RTD could then be considered the frequency distribution of the contributing flow paths, each having a different residence time but all being a Dirac delta distribution.

The main limitation of linear combinations is that the different sub-systems must not interact. In other words, no exchange by diffusion or lateral dispersion must occur. It is nevertheless a powerful and probably underutilized tool. In particular, the outlet of hydrologic systems is often at the convergence of flow paths that have journeyed in fairly different sub-systems with limited exchange, such as in the case of streamflow Steady-state analytical RTDs 
(contribution from direct rainfall, stormflow, baseflow, and upstream flow) and pumping wells (contribution from different hydrogeologic layers or point-sources). The identification of a linear combination of simple models that reasonably approximates a more complex RTD is also an efficient platform for hypothesis testing. It is indeed straightforward to assess sensitivities for various combinations of sub-systems, as all of the components required for these analyses are analytical, and each component is independent of the others.

Another issue with this approach is that the number of parameters can be greatly increased. Each sub-system requires its own distribution parameters - often 2 or 3 - and its weight to be estimated - which gives $N-1$ additional parameters, thus increasing the complexity of the task; for instance, for a mixing of three compartments, this leads to a system of about 10 unknowns $(3 N-1$ to $4 N-1)$. It might also be tempting to increase the number of components in the objective of getting a better fit; this implies the risk of losing the physical meaning of the parameters, and altogether the predictive capabilities of the model.

\subsubsection{Gamma distribution}

An example of parallel mixing is the three-parameter Gamma distribution, which has an exponential form:

$$
\rho_{T}(a)=\frac{(a-\varepsilon)^{\alpha-1}}{\kappa^{\alpha}} \frac{1}{\Gamma(\alpha)} \exp \left(-\frac{a-\varepsilon}{\kappa}\right)
$$

where $\alpha[-]$ is called the shape parameter, $\kappa[\mathrm{T}]$ is called the scale parameter, $\varepsilon[\mathrm{T}]$ is called the location parameter - it may simply correspond to the system lag as in Equation (9) and $\Gamma(\alpha)$ is the Gamma function defined by:

$$
\Gamma(\alpha)=\int_{0}^{+\infty} u^{\alpha-1} \exp (-u) d u
$$

The mean residence time $\tau$ is defined by:

Steady-state analytical RTDs

Page 32 of 77 


$$
\tau=\alpha \kappa+\varepsilon
$$

The Gamma distribution cannot be derived as a single solution of Eq. (2). Accordingly, the physical connection between this distribution and hydrologic systems is not straightforward. However, one can produce a few well-known analytical solutions from specific values of its parameters. For instance, defining the shape parameter $\alpha$ as $1, \Gamma(\alpha)$ is then 1 and the RTD reduces to the exponential-piston model (see section 4.3). If $\varepsilon$ is then defined to be 0 , this further reduces the exponential-piston to the exponential model (Eq. (26)). Clearly, all three distributions belong to the same family. The Gamma is obviously the most flexible: depending on the parameter $\alpha$, the RTD goes from a Gaussian-type to a power-type, including an exponential-type (Figure 7). Though, it is also the most difficult to link to a system's parameters.

Gamma distributions, and other Lumped Parameter Models (LPMs), have seen limited use in groundwater applications compared to their widespread application in catchment hydrology (see McGuire and McDonnell (2006) for a summary of the LPMs used in catchment and subsurface hydrology). The work of Kirchner et al. $(2000 ; 2001)$ is particularly noteworthy as it provided a thorough analysis of the possible origin of the Gamma distribution and of its applicability. A frequency analysis of chloride stream concentrations was used to demonstrate fractal scaling of residence times within a few catchments that could be fitted with the Gamma RTD setting $\alpha$ to 0.5 and $\varepsilon$ to 0 :

$$
\rho_{T}(a)=\sqrt{\frac{1}{2 \pi \tau a}} \exp \left(-\frac{a}{2 \tau}\right)
$$

They concluded that the gamma distribution was well-suited for catchment scale systems as it could model system's response to short-term inputs i.e. the closest ones to the stream as well as long-term ones thanks to a power-law tail, broader than with conventional RTDs. The authors also integrated the one-dimensional advection-dispersion RTD along the entire length $L$ of the hilltop with a uniform recharge $R$ giving an equal weight to each flow path. The following equation is the continuous analog of Eq. (49) using $\rho_{T}(a)$ of Eq. (46) and $R$ for $\lambda_{n}$ ):

Steady-state analytical RTDs 


$$
\rho_{T}(a)=\frac{\int_{x=0}^{L} R \rho_{T}(a) d x}{\int_{x=0}^{L} R d x}=\frac{1}{L} \int_{x=0}^{L} \rho_{T}(a) d x
$$

The RTD obtained was similar in form to the Gamma distribution (see their Eq. 11) for Peclet numbers close to 1. Physical situations where Gamma distributions may be appropriate are when dispersivities are as large as the hillslope length arising from a wide variety of flow paths. Possible mechanisms for that include mass transfer between mobile and immobile zones with long retention times (Russian et al., 2013; Scher et al., 2002), large contrasts of conductivity at all scales resulting in preferential flow (Lindgren et al., 2004), and complex flow patterns caused by local hillslope topography or catchment geometry (Cardenas, 2007; McGuire et al., 2005). The parameter $\alpha$ might be used to specifically express a multi-scale aspect of the transport in the system. The Gamma distribution may be a reasonable model for problems lying beyond the assumptions made in classical LPM (e.g. Dupuit-Forchheimer flow or homogeneity of petrophysical properties). Still, there remains a lack of clear physical mechanisms relating the Gamma to the properties of hydrologic systems and so it is relegated to a descriptive role.

\subsection{Series mixing}

Series mixing is the compliment to parallel combinations in that the system is also decomposed into several independent sub-systems but here they fully and successively interact (Figure 6b). The RTD of a single system represents how the system responds to a specified input, i.e. how water with a distribution of zero residence time is modified through a specific system. For this reason, a single RTD can also be represented as:

$$
\rho_{T}(a)=\int_{0}^{\infty} \delta(a ́) \rho_{T}(a-a ́) d a ́
$$

where $\delta(a)$ is the dirac-delta function and $a ́$ is an integration variable. This equation effectively stipulates that the inflow to the system is of zero residence time. In the case of systems in series, the outflow of a sub-system is assumed to be the inflow of a second sub-system. Assuming further that perfect mixing occurs either in the systems or at their 
interface, the combined RTD of two systems in series can be described by the convolution product as follows (Bracewell, 2000; Press, 2007; Wiener, 1949):

$$
\rho_{T}(a)=\int_{0}^{\infty} \rho_{1}(a ́) \rho_{2}(a-a ́) d a ́
$$

where $\rho_{1}$ is the RTD of the first system and $\rho_{2}$ is the RTD of the second system. This technique can be repeated any number of times. The net effect depends on the properties of each sub-system but is independent of their ordering.

Series mixing models are used in chemical engineering to explain mixing in reactors (e.g. Martin, 2000). The properties of these models can also be used for inverse problems, as demonstrated by Engdahl et al (2013) who showed that the travel time between two points could be constructed from the deconvolution of two RTDs, assuming a series mixing forward model. Mixing cell models derive RTDs as a series combination of perfectly-mixed systems (Campana, 1987). In this case, individual mixing cells are composed of inflows from adjacent cells and recharge. Hence the total RTD arises as a series combination of upstream mixing cells.

An important point is that the variance of the distribution cannot decrease and that the resulting RTD will be no more ordered than its least ordered component. Convolutions are filters that stretch out distributions and the minimum allowable change in variance is zero, which is only possible for a Dirac input function. Any other input function will increase the variance of the combined RTD; note that this is consistent with the natural world because systems do not spontaneously un-mix.

A number of individual processes that act at sub-aquifer or sub-catchment scale give rise to specific RTDs. Examples are the travel time through a hillslope (Fiori and Russo, 2008), exchanges between streams and groundwater (Cardenas, 2008), and transport through aquifers (Weissmann et al., 2002). All these processes are dynamic and exchanges readily occur between different parts of a spatially distributed hydrologic system. Therefore, it is likely that some portion of the water can be approximated as moving between individual compartments, resulting in some level of series mixing. 
Overall, single transfer functions have been the most widely used construction throughout the residence time literature and series mixing models are probably underutilized tools.

\subsection{Lagged systems}

Lagged systems constitute a particular case of a combination of two systems where either the first or the second compartment can be modeled as a piston flow. The response of the combined system appears to be delayed (Levenspiel, 1999; Małoszewski and Zuber, 1982). As the shift $a_{0}[\mathrm{~T}]$ applies to every flow path, the mean residence time of the combined system $\tau_{L}$ includes the additional contribution of the shift:

$$
\tau_{L}=\tau+a_{0}
$$

where $\tau$ is the mean residence time of the non-piston compartment. Lagged systems are clearly scale-dependent (IAEA, 2006): a linear relation binds the mean residence time to the shift $a_{0}$ which is itself is a direct function of the extension of the piston compartment. The lagged RTD $\rho_{L}(a)$ is merely obtained with a change of variables from the non-piston distribution $\rho_{T}(a)$ :

$$
\begin{cases}\rho_{L}\left(a, a_{0}\right)=\rho_{T}\left(a-a_{0}\right), & a \geq a_{0} \\ \rho_{L}\left(a, a_{0}\right)=0, & a<a_{0}\end{cases}
$$

Provided it has a physical meaning, the concept of lagged systems can be very practical as it can be applied whatever the non-piston compartment and hence covers a wide range of configurations. Some combinations of systems fitting in this framework are:

- Semi-confined aquifers recharged by a vertically-leaking aquifer (piston part) provided the sampling does not capture flow lines from the leaking aquifer and that the recharge of the leaking aquifer is uniform (Figure 8a). The upstream shift $a_{0}$ is a direct function of the recharge rate and of the thickness of the leaking aquifer $H_{\text {leak }}$ :

$$
a_{0}=\frac{\theta H_{\text {leak }}}{R}
$$

- Confined aquifers (piston part) recharged by an adjacent unconfined aquifer (e.g. Chen et al., 2011; Ivey et al., 2008). The downstream shift $a_{0}$ is a function of the horizontal 
velocity from the adjacent unconfined aquifer (Figure 8b). In Table 1 are some examples of the shift value depending on the adjacent aquifer properties under purelyadvective conditions.

- Semi-confined or unconfined aquifer (such as in section 3.2.2) whose shortest flow paths are not sampled, e.g. sampling in an observation well which is screened over the bottom part of the system (Małoszewski and Zuber, 1982).

\subsection{Non-Fickian systems}

Another approach to modeling complex RTDs is to revisit the governing equations and consider the conditions under which those partial differential equations are valid. For example, solutions of equation (2) are only valid for a partial differential equation where the underlying dynamic process has reached its asymptotic behavior and does not change further; these assumptions may be violated in real systems where heterogeneity exists at multiple scales. Also, consider the distinction between kinematic porosity and total porosity. Water is continuously diffusing and, just like solute transport, this can include diffusion into dead-end pores, which is an immobile domain. The solute transport literature has termed these as non-Fickian behaviors and it is worthwhile to consider these processes in the context of residence time. The primary features of non-Fickian transport are fast arrivals and/or late time tailing, relative to a Fickian model, for which the RTD analog would be short and long residence times, respectively. The fast arrivals are the result of preferential flow through uncharacterized fast pathways and late time tailing may be caused by mass transfer, back diffusion, or broad ranges of velocities (Benson et al., 2000; Carrera et al., 1998; Haggerty and Gorelick, 1995). Much of this terminology originates from subsurface solute transport literature but similar analogies can be made for other systems.

The theories of non-Fickian and pre-asymptotic solute transport are homogenization approaches that account for the role of heterogeneities, or immobile mass transfer, on solutes, without explicitly resolving those heterogeneities. The body of literature on the subject is vast but a brief perspective with references to the different theories can be found in Neuman and Tartakovsky (2009). Most studies on non-Fickian transport lead to 
a 1-D analytical model for describing RTDs. Still, they often exist as convolutions or in the complex plane, and numerical solution or inversion of integral transforms is then often required.

\subsubsection{Non-Fickian solutions for RTDs}

To date, two analytical models have been presented in the literature that specifically address non-Fickian steady-state RTDs. The first is found in Ginn et al. (2009) and is the modified solution of Lassey (1988) for dual-domain mass transfer applied to residence time. This model assumes reversible, kinetic mass transfer between the mobile and immobile domains in a 1-D semi-infinite problem. The effect of these processes is to stretch out and shift mass into the tail of the distribution, relative to an inverse Gaussian.

The second application of non-Fickian transport to RTDs is the general memory function approach for solute transport (Dentz et al., 2004). Memory functions have seen widespread use in solute transport and are equivalent to the Lassey (1988) model in some cases. The general 1-D analytical solution for a Dirac-delta initial condition is defined through its Laplace transform:

$$
\tilde{\rho}(x, s)=\exp \left[-\frac{x v}{2 D_{l}}\left(\sqrt{1+4 \frac{s D_{l}}{\widetilde{M}(s) v^{2}}}-1\right)\right]
$$

where $\sim$ denotes a Laplace transformed function, $s$ is the Laplace dual to $a, x$ is 1-D position, $D_{l}$ is a longitudinal dispersion coefficient, and $\widetilde{M}(s)$ is the Laplace transformed memory function (Engdahl et al., 2013). The form of the memory function controls the deviation of this model from an inverse Gaussian, which is recovered when $\widetilde{M}(s)=1$.

Other equivalent formulations exist (Silva et al., 2009) such the fractional ADE (Benson et al., 2000; Berkowitz et al., 2002; Schumer et al., 2003), Continuous Time Random Walk, Multi-Rate Mass Transfer (Carrera et al., 1998; Gouze et al., 2008; Haggerty and Gorelick, 1995), and generalized memory function approaches (Cvetkovic, 2012; Silva et al., 2009). The solution presented here is a general non-local in time formulation. This kind of solutions allows greater complexity to be modeled analytically but carry additional cost. The above solution is defined by its Laplace transform and numerical Steady-state analytical RTDs 
inversion of the Laplace transform can be very unstable. The choice of an appropriate memory function has been difficult to justify a priori historically, so these applications have often been restricted to descriptive tasks. However, it is noteworthy that recent developments have started to establish more robust links between geological formations and memory functions, therefore improving the predictive capabilities of these models (Gouze et al., 2008; Russian et al., 2013). The dual-domain solution requires the numerical evaluation of an integral and also carries some additional parameterizations such as mass transfer rates. These solutions are clearly not as straightforward as the solutions of section 3, but, since they assume a physical mechanism for transport, they can be used for forward predictions. The predictions will contain the embedded uncertainty of all the parameters within the models, as well as the assumption that the model is correct. It is also possible to create linear combinations using these non-Fickian techniques but this introduces the additional uncertainty of the flux weights.

\subsubsection{Scale-dependent dispersion model}

Another representation of non-Fickian processes is the scale-dependent dispersion model (Green et al., 2014) which assumes a power-law relationship between the travelled distance $l$ and the anisotropic dispersivity:

$$
\begin{gathered}
D_{l}=\alpha_{l} v_{l}=A l^{B} \\
D_{t}=\frac{D_{l}}{C}
\end{gathered}
$$

where $\alpha_{l}[\mathrm{~L}]$ is the longitudinal dispersivity, $v_{l}$ is the longitudinal velocity, $D_{l}$ and $D_{t}$ are the longitudinal and transverse dispersion coefficients respectively $\left[\mathrm{L}^{2} \mathrm{~T}^{-1}\right], A, B$ and $C$ [-] are constants.

Contrary to the classical solution of transport with longitudinal dispersion presented above, the solution proposed by Green et al. (2014) accounts for transverse dispersion. Hence, the RTD at a sampling point at depth will catch the transport signal of a non-point source of solute (Figure 9). To obtain the RTD, the flux-averaged concentration must 
then be obtained by integration of the concentration over all straight and parallel streamlines entering the aquifer up gradient from the sampling point:

$$
C_{F}(a)=\int_{0}^{\infty} \frac{M}{L} \frac{l}{4 \pi v_{l} a^{2} \sqrt{D_{l} D_{t}}} \exp \left[-\frac{\left(l-v_{l} a\right)^{2}}{4 D_{l} a}-\frac{y^{2}}{4 D_{t} a}\right] d l
$$

where $M$ is the mass of solute per unit length $L$ injected instantaneously $\left[\mathrm{M} \mathrm{L}^{-1}\right]$ and $y$ is the transverse distance which is related to the longitudinal distance $l$ by the relationship:

$$
y=\frac{H_{w} v_{l}-l v_{v}}{v_{h}}
$$

where $v_{h}$ and $v_{v}$ are the velocities in the horizontal and vertical directions respectively and $H_{w}$ is the depth of the sampling point below the water table. The RTD is eventually recovered by dividing the proportion of solute mass with residence time $a$ by the total mass of all residence times:

$$
\rho_{T}(a)=\frac{C_{F}(a)}{\int_{0}^{+\infty} C_{F}(a) d a}
$$

This recent solution is capable of representing heavy-tailed distributions that cannot be reproduced with the classical advection-dispersion solution. While it has a higher number of parameters, these may be estimated from field sampling conditions, which is an advantage over many of the alternative models.

\subsection{General considerations on advanced solutions}

The majority of techniques that can be applied to solute transport of a conserved mass can be applied to residence time, so there are many more possible approaches for modeling non-idealized RTDs. Each model carries its own assumptions, limitations, and data/parameter requirements, along with any implementation or solution stability complications. The flexibility of the advanced solutions can be advantageous but one should consider whether the tradeoffs and uncertainties of a more robust solution are really an improvement over a simpler solution. Complexity for the sake of complexity

Steady-state analytical RTDs

Page 40 of 77 
should be avoided if there is no conceptual basis for its adoption. Other than the nonFickian and scale-dependent solutions, the only pragmatic approach to match real distributions is linear combinations of other analytical solutions. The goodness of fit of composite solutions is then evaluated against tracer data. This comes down to a parameter estimation or optimization problem. In that sense, geochemical and numerical methods are the most adequate methods for advanced study of most "real-world" applications.

\section{On the use of steady-state analytical RTDs}

\subsection{Applicability of the steady-state analytical RTDs}

\subsubsection{System's stationarity}

An important limitation of the models presented in this article is the assumption of constant replenishment. In reality, hydrological systems are dynamic. Temporal variations can be due to natural processes such as the timing and frequency of rainfall in small catchments and long term natural climate change in large aquifer systems. They can also be human induced, through aquifer exploitation or land clearing. These temporal variations in flow will also induce temporal variations in the RTDs of hydrological systems (Duffy, 2010). It is important to note that these variations can be significant, therefore altering the physical interpretations of RTDs if the solution is based on the steady-state assumption (Schwartz et al., 2010). Some of the solutions presented in this paper have also been proposed in transient forms. These include the piston flow model (Ozyurt and Bayari, 2005), the exponential model (Nir, 1973; Ozyurt and Bayari, 2005) and the solution to the one-dimensional advection-dispersion equation (Soltani and Cvetkovic, 2013). The assumptions that underpin these solutions can also be generalised as "equivalent steady-state" or "stretched residence time" models (Ali et al., 2014; Massoudieh, 2013). The solution of RTDs under transient forcing presents a number of different challenges, and subsequent limitations. For this reason a separate paper in this issue has been dedicated to this subject. 


\subsubsection{Amount of fitting parameters}

While complex solutions can be used to represent a large number of systems, some caution must be taken as the assumptions of the solutions might still be invalid. If this is so, the physical interpretations as well as the predictive capabilities of the solution could be seriously hampered. For example, parallel combinations assume no interaction between systems whereas series models represent complete interaction. The reality is that these types of scenarios are only met in very specific circumstances. The incorporation of multiple solutions also requires the identification of individual systems and their parameterisation. This may result in significant uncertainty, or overfitting, where the parameters are unlikely to represent any physical reality. This may limit the predictive capability of such solutions. Further, the use of solutions without a physical basis, such as the Gamma distribution, may also lend themselves to overfitting also limiting predictive capability. The limitations may be overcome by ensuring that solutions are physically valid. In the case of solutions without a physical meaning, relationships may be formed between parameters and measurable quantities (Hrachowitz et al., 2010; Kirchner et al., 2001).

\subsubsection{Spatial variability of petrophysical properties}

Most of the models represented here assume that the physical properties of systems are spatially constant. Properties such as permeability or porosity have significant spatial variability. The use of overly simplistic analytical solutions represents a structural error in that the system is inadequately represented by the solution and the parameters. Ideally the best way to represent the complexities of hydrological systems is through the use of distributed models. Numerical models provide the flexibility to represent spatially variable aquifer properties, in addition to transient forcing. Numerical methods to simulate RTDs include Lagrangian (Weissmann et al., 2002) and Eulerian techniques (Varni and Carrera, 1998)(Cornaton and Perrochet, 2006; Ginn, 1999). Despite the added flexibility of these methods, their use requires a significantly higher level of parameterisation. Their development requires a lot more work, and numerical simulation can be computationally expensive. Both approaches (analytical and distributed models) are in fact complimentary in that analytical can provide a rapid approximation of RTDs Steady-state analytical RTDs 
while distributed models allow for a greater level of details to be considered when required.

\subsection{Experience with steady-state analytical RTDs: uncertainty and non- uniqueness}

The hydrological literature abounds with studies based on analytical solutions for RTDs: (Bockgård et al., 2004; Chen et al., 2011; Knowles et al., 2010; Manning and Caine, 2007; Osenbrück et al., 2006; Solomon et al., 2010; Zouari et al., 2011) for a few recent examples. Since there is no practical way to empirically measure residence time distributions, a commonly used approach is parametric fitting of trial distributions to chemical concentrations (Turnadge and Smerdon, 2014). This allows examination of the validity of an assumed model while informing about the time past since water has entered the system. Typically used tracers either have a known input function $c_{i n}$ such as historic atmospheric concentration records for $\mathrm{CFCs}, \mathrm{SF}_{6}$, or have a known decay or production rate (with decay constant $\lambda$ ) in the system such as ${ }^{14} \mathrm{C}$, ${ }^{39} \mathrm{Ar}$, or both like ${ }^{85} \mathrm{Kr},{ }^{3} \mathrm{H},{ }^{3} \mathrm{He}$. In practice, the procedure is often very similar: the concentrations $c_{\text {out }}$ observed at time $t$ are estimated from the convolution of the assumed analytical model for the RTD with the tracer's input function $c_{i n}$ :

$$
c_{\text {out }}(t)=\int_{0}^{+\infty} c_{\text {in }}(t-a) \rho_{T}(a) \exp (-\lambda a) d a
$$

Prospective RTDs that give consistent reproduction of the data $c_{\text {out }}(t)$ are selected and some predictions may be finally attempted. To that end, several tools have been developed to match solutions of RTDs to tracers data (Bayari, 2002; Fienen et al., 2006; Suckow, 2012; Tang et al., 2012) and some studies go so far as to propose analytical solutions to equation (66) using synthetic functions for $c_{\text {in }}$ (Jódar et al., 2014). Note that equation (66) is not limited to analytical expressions for $\rho_{T}(a)$ and is valid whatever the method used to generate the RTD including numerical simulation.

However, it is not uncommon to find a few distinct models that provide consistent and similar results. Indeed, the details of the RTD are lost when convoluting the tracer's input 
function with the RTD; what remains is only a weighted concentration which encapsulates information from both the RTD and the temporal variations of the tracer input function. Another constraint to this is that a tracer only captures the part of the RTD that corresponds to its temporal occurrence. Tracers can actually be seen as blurred snapshots of the RTD. Finally, some analytical solutions can appear very similar in shape depending on the value of their parameters which is another limiting factor: it can be the case of the dispersive model and the exponential-piston (Marçais et al., 2015). It may also be the case that different tracer input functions look very alike (e.g. CFCs, Leray et al., 2014) and then give redundant information about the RTD. When data series of tracers are available, the interpretation of its temporal evolution is besides subject to the decoupling of the evolution of the tracer's input function and potential temporal variations of the system's RTD (Leray et al., 2014).

With these precautions in mind, it is important to consider that consistency is no guarantee of accuracy, or even correctness. For example, a model that reproduces concentrations but does not adequately characterize the flow field will fail when used for prediction on other time scales - typically on lower time scales (Leray et al., 2012). Thus, the consistency of models is necessary but not sufficient (Eberts et al., 2012). The best example of this is the piston-flow model which is very often consistent but also mainly non-predictive. Gaining an understanding of flow from tracer data is then far from obvious and the critical step in this process remains the choice of a presumed RTD, which must reflect the physical functioning of the system for a meaningful interpretation.

While assessing the uncertainty associated with the shape of the RTD is a hard task, quantifying uncertainty for the tracers is more feasible. The multiple source of uncertainties include those in the measured tracer concentrations both as a result of analytical errors or lack of representativeness due to spatial and temporal heterogeneities, uncertainties associated with the tracer's input function $c_{i n}$, and in some cases the fact that the decay or production rate of tracers in the system is unknown or uncertain. In order to use inferred RTDs to assess watershed management strategies, assess the vulnerability of water resources or evaluate the sustainability or water withdrawals, it is important to rigorously translate these sources of uncertainty into the uncertainty of the 
inferred parameters of the RTD. One way to do this is to use backward uncertainty propagation methods. Several researchers have used Bayesian inference to perform uncertainty assessment on the parameters of RTDs (Cirpka et al., 2007; Massoudieh et al., 2012; Payn et al., 2008). The Bayesian approach provides an estimated joint probability distribution of the RTD parameters instead of point estimates and therefore can reveal non-uniqueness, parameter correlation and information content of each of the tracers.

Although non-uniqueness and uncertainty are a limitation of LPMs, most hydrological techniques, including numerically-simulated RTDs, possess the same limitation. The best way to deal with this is through rigorous comparison and the incorporation of informative data. Analytical solutions for the RTD remain a powerful tool as they constitute a clear, fast and easily-accessible interface between observations and physical functioning of real hydrologic systems. When there is no a priori knowledge on the flow pattern, these solutions can be included in an iterative process in which they can help for uncertainty assessment and acquisition of additional data. In some cases, additional data may help to constrain the parameters of specific model structures, or reduce the number of potential model structures. Besides, alternative methods such as non-parameterized RTDs or the concept of the export rate affinity with time exist that give even more flexibility (see section 5.3).

\subsection{Alternatives for physically-based RTDs}

\subsubsection{Export rate affinity with residence time}

In some complex hydrological systems for example in catchment hydrology, describing the movement of water using an advection-dispersion transport models or other processbased approaches is challenging due to large differences in flow pathway time-scales (e.g. surface runoff, vadose zone and base-flow, evaporation) and high levels of heterogeneity. In such cases what can be done is to treat the entire system as a single reservoir while considering the outflow rate to be function of the residence time (Botter et al., 2010; Botter et al., 2011). This method has been developed for transient systems but equally applies to steady-state ones. It should be noted that this is not strictly a 
physically-based approach but since the water export is connected to the residence time of water in a system, information from the overall physical hydrological behavior can be incorporated into the function relating the outflow rate and residence time.

A simple relation between the outflow rate of water $Q_{\text {out }}(a)$ and the exiting residence time distribution $\rho_{T}(a)$ would be $Q_{o u t}(a)=\rho_{T}(a) \int_{0}^{\infty} Q_{o u t}(a) d a$ as in Equation (17) (Etcheverry and Perrochet, 2000). However, Botter et al. (2011) proposed a multiplier $\beta(a)$ to include a specific relationship (or an affinity) between the residence time distribution of the exiting water and the in situ residence time distribution resulting in:

$$
Q_{\text {out }}(a)=\beta(a) \rho_{T}(a) \int_{0}^{\infty} Q_{\text {out }}(a) d a
$$

The cumulative residence time distribution $\mathrm{P}_{w}(a)$ is equally marked by the affinity. Its analog, noted $\gamma\left[\mathrm{P}_{w}(a)\right]$ in this framework, has been successively called "Age function", "Storage Outflow Probability", and lately, the cumulative of the StorAge Selection (SAS) function (Harman, 2015; Queloz et al., 2015; van der Velde et al., 2012). It is defined by:

$$
\gamma\left[\mathrm{P}_{w}(a)\right]=\int_{0}^{a} \beta(a) \rho_{w}(a) d a
$$

The SAS function and its cumulative form lump system's characteristics and the mixing effects within the system. When used in transient conditions, they further include the effect of transient boundary conditions on the RTD. Their form is at one's discretion with the mere exception that $\int_{0}^{\infty} \beta(a) \rho_{w}(a) d a=1$ for water balance equation to hold. The multiplier $\beta(a)$ can take the form of a uniform function (no affinity with the export rate, $\gamma\left[\mathrm{P}_{w}\right]=\mathrm{P}_{w}$ ), a Dirac delta distribution (affinity to one residence time only, similar to the piston flow) or any other function, linear or not. The cumulative SAS function $\gamma\left[\mathrm{P}_{w}\right]=$ $\mathrm{P}_{w}{ }^{n}$ nicely illustrates the flexibility of the method presented here: an exponent $n>1$ means a higher export affinity towards older residence times compared to younger residence times, and inversely, an exponent $n<1$ indicates a larger tendency of export from younger residence time compared to older residence times. This approach Steady-state analytical RTDs 
constitutes an appealing alternative for preliminary study of systems in which none of the physically-based RTDs can be clearly preferred.

\subsubsection{Shape-free methods}

Another alternative to physically based LPMs is the use of shape-free methods (Cirpka et al., 2007; Fienen et al., 2006; Liao et al., 2014; Luo and Cirpka, 2008; Massoudieh et al., 2014; McCallum et al., 2014; Payn et al., 2008; Visser et al., 2013). These recent techniques assume no specific form of the RTD but rather consider RTDs as histograms with given number of uniform on non-uniformly sized bins and then the fractions in each bin is treated as unknown parameters which are estimated using tracer data. As this is primarily a data-driven technique, the detail of the RTD can be limited by the amount and type of data. Another challenge is that when the number of bins is large compared to the amount of tracer data, the problem can be over-parameterized and therefore unidentifiable. One way of dealing with this is the use of relatively coarse compartments (Massoudieh et al., 2014; Visser et al., 2013). Alternatively, large numbers of compartments can be used in conjunction with forms of regularization or by imposing constraints on the degree the adjacent bin fractions can be different (Cirpka et al., 2007; Fienen et al., 2006; Luo and Cirpka, 2008) or numerical regularization (McCallum et al., 2014). However, the main limitation of these methods is an ample amount of data and sufficient variability in the input concentrations - that is enough to characterize each compartment. These methods are advantageous when the form of the distribution is the primary interest of the study.

\section{Future directions}

A wide range of analytical solutions for RTDs exist and it is worth taking a moment to ponder what remains. All of the analytical models here assume a simple system, or simplify the mechanisms within a flow system, yet the evidence in the literature shows that heterogeneity can play a significant role. Adaptation of the simple models to include non-ideal behavior is an important step toward generalizing the models but this has been explored for decades by the solute transport community. Considering more complex physical configurations such as the effect of near groundwater divide or simple variations 
of system's features, is another step forward. The limitation has always been, and continues to be, identifying relationships between the observable features of complex natural systems and the bulk parameters in the RTD models; this is the case for the Gamma distribution whose origin seems close to classical simpler solutions but which is not clearly demonstrated. Numerical simulation can besides help in that matter. This is still a meaningful endeavor and merits additional work given the high cost and uncertainty that can be associated with the alternative distributed parameter models.

Another, relatively unexplored, component of residence time is the effect of transience. Most systems encountered in nature are in fact under some degree of change. This can be due to long-term climate changes, seasonality, variations in forcing or significant alterations of systems. Ultimately these dynamic changes may have impacts on the shape of RTDs. Anytime a steady-state model is applied, it assumes that the long-term behavior of the system does not change over time but, as we have seen, the mathematics are considerably simplified. Deviations of an RTD from an analytical model could then reflect poor model choice but whether this stems from heterogeneity or transience may be difficult to determine. The validity of the steady-state approximation is seldom considered, but it may emerge as a critical element. As fewer and fewer hydrologic systems in the world remain undisturbed mainly due to human activities, the role of transience in determining the shape of RTDs is a continuing area of research (Botter et al., 2011; Cornaton, 2012; Gomez and Wilson, 2013). This issue of transience is significant enough that a companion paper in this volume specifically addresses the state of transient RTD modeling.

In this paper we have presented a number of solutions of RTDs. It is interesting to examine how studies of RTDs have evolved in order to assess the future directions in this area. Early solutions of RTD were based on the assumption of simple flow and/or mixing processes (e. g. solutions presented in section 3). Often the aim behind this type of solution was to determine a physical meaning associated with the parameterization of a specific model (e.g. a recharge rate or a mean transit time). However, field studies (e.g. Kirchner et al., 2000) began to demonstrate a discrepancy between RTDs expected from simple solutions of residence time, and those that could explain concentration data 
obtained from real systems. Further, the development of numerical methods also demonstrated that physical heterogeneity and variable recharge-discharge relationships produced RTDs which deviated from what was expected from simple models.

Attempts to reconcile these apparent differences have led to the development of more complex solutions. One set of complex solutions arose from the combination of existing simple solutions. Others have attempted to include more complex mixing processes. Others still have removed a physical basis and simply aim to represent complex distributions (e.g. power law and Gamma distributions). This rise of complex solutions has also accompanied a shift in the focus of these solutions. Rather than attempting to parameterize a simple flow system, RTDs began to represent transfer functions for the movement of solutes through systems as the concentration data used to infer RTDs moves through systems as solutes and are susceptible to many of the same phenomena observed in contaminant transport studies. This meant that there was a greater need to accurately represent the shape of the RTD, rather than the physical meaning of the system that it came from.

Given the current state of RTD research, there are a number of areas that still offer potential for significant advances. Firstly, an understanding of if and when simple solutions are appropriate can be extended. Current examples include work by Eberts et al. (2012), Green et al. (2014) and Marçais et al. (2015). This and further work provide guidance for the use of simple solutions, which may be implemented with small amounts of data and limited understanding of the physical system. Secondly, understanding the mechanisms that result in the observed heavy-tailing and early arrivals times that seem consistent across many observations should continue. Some current work has related this to work on solute transport in heterogeneous systems (Engdahl et al., 2012; Green et al., 2014; Scher et al., 2002) and flow system dynamics at multiple scales (Cardenas, 2008; Kirchner et al., 2001). However, these studies by no means constitute an exhaustive list of mechanisms that can influence RTDs. Finally, the practice on inferring the shape of RTDs offers some potential areas for improvement. Often this technique relies on concentration data. The type and amount of data is often dependent on the time scales covered by the RTD. At very small residence times of interest to water quality (e.g. hours 
- days) natural tracers such as temperature and electrical conductivity, or applied tracer tests may be available. At much larger scales of interest to aquifer management the only data available may be from radioactive isotopes. This limits the level of detail that can be obtained about the RTD. Another consideration for the use of concentration data is the limit of RTDs to represent certain reaction. In a general sense, only concentrations dependent on zero and first order reactions can be predicted by RTDs. This limits the general utility of RTDs as transfer functions to predict contaminant concentrations.

\section{References}

Ali, M., Fiori, A., Russo, D., 2014. A comparison of travel-time based catchment transport models, with application to numerical experiments. Journal of Hydrology, 511(0): 605-618. DOI:http://dx.doi.org/10.1016/j.jhydrol.2014.02.010

Amin, I.E., Campana, M.E., 1996. A general lumped parameter model for the interpretation of tracer data and transit time calculation in hydrologic systems. Journal of Hydrology, 179(1-4): 1-21. DOI:http://dx.doi.org/10.1016/0022$\underline{1694(95) 02880-3}$

Bayari, S., 2002. TRACER: an EXCEL workbook to calculate mean residence time in groundwater by use of tracers CFC-11, CFC-12 and tritium. Computers \& Geosciences, 28(5): 621-630. DOI:http://dx.doi.org/10.1016/S0098$\underline{3004(01) 00094-2}$

Bear, J., 1972. Dynamics of Fluids in Porous Media. Dover Classics of Science and Mathematics. Dover.

Begemann, F., Libby, W.F., 1957. Continental water balance, ground water inventory and storage times, surface ocean mixing rates and world-wide water circulation patterns from cosmic-ray and bomb tritium. Geochimica et Cosmochimica Acta, 12(4): 277-296. DOI:http://dx.doi.org/10.1016/0016-7037(57)90040-6

Benettin, P., Rinaldo, A., Botter, G., 2013. Kinematics of age mixing in advectiondispersion models. Water Resources Research, 49(12): 8539-8551. DOI:http://dx.doi.org/10.1002/2013wr014708

Benson, D.A., Wheatcraft, S.W., Meerschaert, M.M., 2000. The fractional-order governing equation of Lévy Motion. Water Resources Research, 36(6): 14131423. DOI:http://dx.doi.org/10.1029/2000wr900032 
Berkowitz, B., Klafter, J., Metzler, R., Scher, H., 2002. Physical pictures of transport in heterogeneous media: Advection-dispersion, random-walk, and fractional derivative formulations. Water Resources Research, 38(10): 9-1-9-12. DOI:10.1029/2001wr001030

Bethke, C.M., Johnson, T.M., 2002. Paradox of groundwater age: Correction1. Geology, 30(4): 385-388. DOI:http://dx.doi.org/10.1130/00917613(2002)030<0386:POGAC>2.0.CO;2

Bethke, C.M., Johnson, T.M., 2008. Groundwater Age and Groundwater Age Dating. Annual Review of Earth and Planetary Sciences, 36(1): 121-152. DOI:http://dx.doi.org/10.1146/annurev.earth.36.031207.124210

Beven, K.J., 2010. Preferential flows and travel time distributions: defining adequate hypothesis tests for hydrological process models. Hydrological Processes, 24(12): 1537-1547. DOI:http://dx.doi.org/10.1002/hyp.7718

Bockgård, N., Rodhe, A., Olsson, K.A., 2004. Accuracy of CFC groundwater dating in a crystalline bedrock aquifer: Data from a site in southern Sweden. Hydrogeol J, 12(2): 171-183. DOI:http://dx.doi.org/10.1007/s10040-004-0319-3

Botter, G., Bertuzzo, E., Rinaldo, A., 2010. Transport in the hydrologic response: Travel time distributions, soil moisture dynamics, and the old water paradox. Water Resources Research, 46(3). DOI:http://dx.doi.org/10.1029/2009WR008371

Botter, G., Bertuzzo, E., Rinaldo, A., 2011. Catchment residence and travel time distributions: The master equation. Geophysical Research Letters, 38(11): n/a-n/a. DOI:http://dx.doi.org/10.1029/2011gl047666

Bracewell, R.N., 2000. The Fourier Transform and Its Applications. Electrical engineering series. McGraw Hill.

Burbery, L.F., Flintoft, M.J., Close, M.E., 2013. Application of the re-circulating tracer well test method to determine nitrate reaction rates in shallow unconfined aquifers. Journal of contaminant hydrology, 145: 1-9. DOI:http://dx.doi.org/10.1016/j.jconhyd.2012.11.006

Burton, W.C., Plummer, L.N., Busenberg, E., Lindsey, B.D., Gburek, W.J., 2002. Influence of Fracture Anisotropy on Ground Water Ages and Chemistry, Valley and Ridge Province, Pennsylvania. Ground Water, 40(3): 242-257. DOI:http://dx.doi.org/10.1111/j.1745-6584.2002.tb02652.x

Campana, M.E., 1987. Generation of Ground-Water Age Distributions. Ground Water, 25(1): 51-58. DOI:http://dx.doi.org/10.1111/j.1745-6584.1987.tb02115.x 
Cardenas, M.B., 2007. Potential contribution of topography-driven regional groundwater flow to fractal stream chemistry: Residence time distribution analysis of Tóth flow. Geophysical Research Letters, 34(5): n/a-n/a. DOI:http://dx.doi.org/10.1029/2006gl029126

Cardenas, M.B., 2008. Surface water-groundwater interface geomorphology leads to scaling of residence times. Geophysical Research Letters, 35(8): n/a-n/a. DOI:http://dx.doi.org/10.1029/2008g1033753

Carrera, J. et al., 1998. On matrix diffusion: formulations, solution methods and qualitative effects. Hydrogeol J, 6(1): 178-190. DOI:http://dx.doi.org/10.1007/s100400050143

Carrera, J., Varni, M., 2000. On the use of environmental tracers to characterize flow and transport parameters. CRC Press, pp. 467.

DOI:http://dx.doi.org/10.1029/98WR02536

Castro, M.C., Goblet, P., Ledoux, E., Violette, S., de Marsily, G., 1998. Noble gases as natural tracers of water circulation in the Paris Basin: 2. Calibration of a groundwater flow model using noble gas isotope data. Water Resources Research, 34(10): 2467-2483. DOI:http://dx.doi.org/10.1029/98wr01957

Chen, Z., Wei, W., Liu, J., Wang, Y., Chen, J., 2011. Identifying the recharge sources and age of groundwater in the Songnen Plain (Northeast China) using environmental isotopes. Hydrogeol J, 19(1): 163-176.

DOI:http://dx.doi.org/10.1007/s10040-010-0650-9

Cirpka, O.A. et al., 2007. Analyzing Bank Filtration by Deconvoluting Time Series of Electric Conductivity. Ground Water, 45(3): 318-328.

DOI:http://dx.doi.org/10.1111/j.1745-6584.2006.00293.x

Cirpka, O.A., Kitanidis, P.K., 2001. Travel-Time Based Model of Bioremediation Using Circulation Wells. Ground Water, 39(3): 422-432.

DOI:http://dx.doi.org/10.1111/j.1745-6584.2001.tb02326.x

Clement, T.P., Truex, M.J., Hooker, B.S., 1997. Two-Well Test Method for Determining Hydraulic Properties of Aquifers. Ground Water, 35(4): 698-703.

DOI:http://dx.doi.org/10.1111/j.1745-6584.1997.tb00136.x

Cook, P., Böhlke, J.-K., 2000. Determining Timescales for Groundwater Flow and Solute Transport. In: Cook, P., Herczeg, A. (Eds.), Environmental Tracers in Subsurface Hydrology. Springer US, pp. 1-30. DOI:http://dx.doi.org/10.1007/978-1-4615$\underline{4557-6 \_1}$

Cornaton, F., Perrochet, P., 2006. Groundwater age, life expectancy and transit time distributions in advective-dispersive systems: 1. Generalized reservoir theory.

Steady-state analytical RTDs

Page 52 of 77 
Advances in Water Resources, 29(9): 1267-1291.

DOI:http://dx.doi.org/10.1016/j.advwatres.2005.10.009

Cornaton, F.J., 2012. Transient water age distributions in environmental flow systems: The time-marching Laplace transform solution technique W03524. Water Resources Research, 48(3). DOI:http://dx.doi.org/10.1029/2011wr010606

Cvetkovic, V., 2012. A general memory function for modeling mass transfer in groundwater transport. Water Resources Research, 48(4): n/a-n/a.

DOI:10.1029/2011wr011657

Danckwerts, P.V., 1953. Continuous flow systems. Distribution of residence times. Chemical Engineering Science, 2: 13. DOI:http://dx.doi.org/10.1016/00092509(96)81810-0

Delhez, E.J.M., Campin, J.-M., Hirst, A.C., Deleersnijder, E., 1999. Toward a general theory of the age in ocean modelling. Ocean Modelling, 1(1): 17-27. DOI:http://dx.doi.org/10.1016/S1463-5003(99)00003-7

Dentz, M., Cortis, A., Scher, H., Berkowitz, B., 2004. Time behavior of solute transport in heterogeneous media: transition from anomalous to normal transport. Advances in Water Resources, 27(2): 155-173. DOI:http://dx.doi.org/10.1016/j.advwatres.2003.11.002

Duffy, C.J., 2010. Dynamical modelling of concentration-age-discharge in watersheds. Hydrological Processes, 24(12): 1711-1718. DOI:http://dx.doi.org/10.1002/hyp.7691

Eberts, S.M., Böhlke, J.K., Kauffman, L.J., Jurgens, B.C., 2012. Comparison of particletracking and lumped-parameter age-distribution models for evaluating vulnerability of production wells to contamination. Hydrogeol J, 20(2): 263-282. DOI:http://dx.doi.org/10.1007/s10040-011-0810-6

Engdahl, N.B., Ginn, T.R., Fogg, G.E., 2012. Non-Fickian dispersion of groundwater age. Water Resources Research, 48(7). DOI:http://dx.doi.org/10.1029/2012wr012251

Engdahl, N.B., Ginn, T.R., Fogg, G.E., 2013. Using groundwater age distributions to estimate the effective parameters of Fickian and non-Fickian models of solute transport. Advances in Water Resources, 54: 11-21.

DOI:http://dx.doi.org/10.1016/j.advwatres.2012.12.008

Engdahl, N.B., Maxwell, R.M., 2014. Approximating groundwater age distributions using simple streamtube models and multiple tracers. Advances in Water Resources, 66(0): 19-31. DOI:http://dx.doi.org/10.1016/j.advwatres.2014.02.001

Steady-state analytical RTDs 
Engdahl, N.B., Maxwell, R.M., 2015. Quantifying changes in age distributions and the hydrologic balance of a high-mountain watershed from climate induced variations in recharge. Journal of Hydrology, 522(0): 152-162.

DOI:http://dx.doi.org/10.1016/j.jhydrol.2014.12.032

Eriksson, E., 1958. The Possible Use of Tritium' for Estimating Groundwater Storage. Tellus, 10(4): 472-478. DOI:http://dx.doi.org/10.1111/j.2153$\underline{3490.1958 . t b 02035 . x}$

Etcheverry, D., 2001. Une approche déterministe des distributions des temps de transit de l'eau souterraine par la théorie des réservoirs - PhD thesis, University of Neuchâtel, Neuchâtel, Switzerland, 118 pp.

Etcheverry, D., Perrochet, P., 2000. Direct simulation of groundwater transit-time distributions using the reservoir theory. Hydrogeol J, 8(2): 200-208. DOI:http://dx.doi.org/10.1007/s100400050006

Fienen, M.N., Luo, J., Kitanidis, P.K., 2006. A Bayesian geostatistical transfer function approach to tracer test analysis. Water Resources Research, 42(7): n/a-n/a. DOI:http://dx.doi.org/10.1029/2005wr004576

Fiori, A., Russo, D., 2008. Travel time distribution in a hillslope: Insight from numerical simulations. Water Resources Research, 44(12): n/a-n/a.

DOI:http://dx.doi.org/10.1029/2008wr007135

Ginn, T.R., 1999. On the distribution of multicomponent mixtures over generalized exposure time in subsurface flow and reactive transport: Foundations, and formulations for groundwater age, chemical heterogeneity, and biodegradation. Water Resources Research, 35(5): 1395-1407. DOI:http://dx.doi.org/10.1029/1999wr900013

Ginn, T.R., Haeri, H., Massoudieh, A., Foglia, L., 2009. Notes on groundwater age in forward and inverse modeling. Transport in porous media, 79(1): 117-134. DOI:http://dx.doi.org/10.1007/s11242-009-9406-1

Goderniaux, P., Davy, P., Bresciani, E., de Dreuzy, J.-R., Le Borgne, T., 2013. Partitioning a regional groundwater flow system into shallow local and deep regional flow compartments. Water Resources Research, 49(4): 2274-2286. DOI:http://dx.doi.org/10.1002/wrcr.20186

Gomez, J.D., Wilson, J.L., 2013. Age distributions and dynamically changing hydrologic systems: Exploring topography-driven flow. Water Resources Research, 49(3): 1503-1522. DOI:http://dx.doi.org/10.1002/wrcr.20127

Gouze, P., Melean, Y., Le Borgne, T., Dentz, M., Carrera, J., 2008. Non-Fickian dispersion in porous media explained by heterogeneous microscale matrix

Steady-state analytical RTDs 
diffusion. Water Resources Research, 44(11): n/a-n/a.

DOI:http://dx.doi.org/10.1029/2007wr006690

Green, C.T., Zhang, Y., Jurgens, B.C., Starn, J.J., Landon, M.K., 2014. Accuracy of travel time distribution (TTD) models as affected by TTD complexity, observation errors, and model and tracer selection. Water Resources Research, 50(7): 6191-6213. DOI:http://dx.doi.org/10.1002/2014wr015625

Grove, D.B., Beetem, W.A., 1971. Porosity and Dispersion Constant Calculations for a Fractured Carbonate Aquifer Using the Two Well Tracer Method. Water Resources Research, 7(1): 128-134. DOI:http://dx.doi.org/10.1029/WR007i001p00128

Haggerty, R., Gorelick, S.M., 1995. Multiple-Rate Mass Transfer for Modeling Diffusion and Surface Reactions in Media with Pore-Scale Heterogeneity. Water Resources Research, 31(10): 2383-2400. DOI:http://dx.doi.org/10.1029/95wr10583

Haggerty, R., Wondzell, S.M., Johnson, M.A., 2002. Power-law residence time distribution in the hyporheic zone of a 2nd-order mountain stream. Geophysical Research Letters, 29(13): 18-1-18-4. DOI:http://dx.doi.org/10.1029/2002gl014743

Haitjema, H.M., 1995. On the residence time distribution in idealized groundwatersheds. Journal of Hydrology, 172(1-4): 127-146. DOI:http://dx.doi.org/10.1016/0022$\underline{1694(95) 02732-5}$

Harman, C.J., 2015. Time-variable transit time distributions and transport: Theory and application to storage-dependent transport of chloride in a watershed. Water Resources Research, 51(1): 1-30. DOI:http://dx.doi.org/10.1002/2014wr015707

Hrachowitz, M., Soulsby, C., Tetzlaff, D., Malcolm, I.A., Schoups, G., 2010. Gamma distribution models for transit time estimation in catchments: Physical interpretation of parameters and implications for time-variant transit time assessment. Water Resources Research, 46(10): n/a-n/a.

DOI:10.1029/2010wr009148

IAEA, 2006. Use of chlorofluorocarbons in hydrology : a guidebook. International Atomic Energy Agency, Vienna, 227 pp.

Ivey, S., Gentry, R., Larsen, D., Anderson, J., 2008. Inverse Application of AgeDistribution Modeling Using Environmental Tracers H3/He3. Journal of Hydrologic Engineering, 13(11): 1002-1010. DOI:http://dx.doi.org/10.1061/(asce)1084-0699(2008)13:11(1002)

Jódar, J., Lambán, L.J., Medina, A., Custodio, E., 2014. Exact analytical solution of the convolution integral for classical hydrogeological lumped-parameter models and

Steady-state analytical RTDs 
typical input tracer functions in natural gradient systems. Journal of Hydrology, 519, Part D: 3275-3289. DOI:http://dx.doi.org/10.1016/j.jhydrol.2014.10.027

Kirchner, J.W., Feng, X., Neal, C., 2000. Fractal stream chemistry and its implications for contaminant transport in catchments. Nature, 403(6769): 524-527. DOI:http://www.nature.com/nature/journal/v403/n6769/suppinfo/403524a0 S1.ht $\underline{\mathrm{ml}}$

Kirchner, J.W., Feng, X., Neal, C., 2001. Catchment-scale advection and dispersion as a mechanism for fractal scaling in stream tracer concentrations. Journal of Hydrology, 254(1-4): 82-101. DOI:http://dx.doi.org/10.1016/S00221694(01)00487-5

Knowles, L., Jr., Katz, B., Toth, D., 2010. Using multiple chemical indicators to characterize and determine the age of groundwater from selected vents of the Silver Springs Group, central Florida, USA. Hydrogeol J, 18(8): 1825-1838. DOI:http://dx.doi.org/10.1007/s10040-010-0669-y

Kollet, S.J., Maxwell, R.M., 2008. Demonstrating fractal scaling of baseflow residence time distributions using a fully-coupled groundwater and land surface model. Geophysical Research Letters, 35(7). DOI:http://dx.doi.org/10.1029/2008GL033215

Kreft, A., Zuber, A., 1978. On the physical meaning of the dispersion equation and its solutions for different initial and boundary conditions. Chemical Engineering Science, 33(11): 1471-1480. DOI:http://dx.doi.org/10.1016/0009-2509(78)85196$\underline{3}$

Lassey, K.R., 1988. Unidimensional solute transport incorporating equilibrium and rate-limited isotherms with first-order loss: 1. Model conceptualizations and analytic solutions. Water Resources Research, 24(3): 343-350. DOI:http://dx.doi.org/10.1029/WR024i003p00343

Lenda, A., Zuber, A., 1970. Tracer dispersion in groundwater experiments, Isotope Hydrology 1970. IAEA, Vienna, pp. 619-641.

Leray, S. et al., 2014. Temporal evolution of age data under transient pumping conditions. Journal of Hydrology, 511: 555-566. DOI:http://dx.doi.org/10.1016/j.jhydrol.2014.01.064

Leray, S., de Dreuzy, J.R., Bour, O., Labasque, T., Aquilina, L., 2012. Contribution of age data to the characterization of complex aquifers. Journal of Hydrology, 464465(0): 54-68. DOI:http://dx.doi.org/10.1016/j.jhydrol.2012.06.052 
Levenspiel, O., 1999. Compartment Models. In: Sons, W.a. (Ed.), Chemical reaction engineering. Wiley Eastern Limited, USA, pp. 10.

Liao, Z., Osenbrück, K., Cirpka, O.A., 2014. Non-stationary nonparametric inference of river-to-groundwater travel-time distributions. Journal of Hydrology, 519, Part D: 3386-3399. DOI:http://dx.doi.org/10.1016/j.jhydrol.2014.09.084

Lindgren, G.A., Destouni, G., Miller, A.V., 2004. Solute transport through the integrated groundwater-stream system of a catchment. Water Resources Research, 40(3): n/a-n/a. DOI:http://dx.doi.org/10.1029/2003wr002765

Long, A.J., Putnam, L.D., 2006. Translating CFC-based piston ages into probability density functions of ground-water age in karst. Journal of Hydrology, 330(3-4): 735-747. DOI:http://dx.doi.org/10.1016/j.jhydrol.2006.05.004

Long, A.J., Putnam, L.D., 2009. Age-distribution estimation for karst groundwater: Issues of parameterization and complexity in inverse modeling by convolution. Journal of Hydrology, 376(3-4): 579-588. DOI:http://dx.doi.org/10.1016/j.jhydrol.2009.07.064

Luo, J., Cirpka, O.A., 2008. Traveltime-based descriptions of transport and mixing in heterogeneous domains. Water Resources Research, 44(9): n/a-n/a. DOI:http://dx.doi.org/10.1029/2007wr006035

Luo, J., Dentz, M., Cirpka, O.A., Kitanidis, P.K., 2007. Breakthrough curve tailing in a dipole flow field. Water Resources Research, 43(9): n/a-n/a. DOI:http://dx.doi.org/10.1029/2006wr005600

Luo, J., Kitanidis, P.K., 2004. Fluid residence times within a recirculation zone created by an extraction-injection well pair. Journal of Hydrology, 295(1-4): 149-162. DOI:http://dx.doi.org/10.1016/j.jhydrol.2004.03.006

Luo, J. et al., 2006. A Nested-Cell Approach for In Situ Remediation. Ground Water, 44(2): 266-274. DOI:http://dx.doi.org/10.1111/j.1745-6584.2005.00106.x

Luther, K.H., Haitjema, H.M., 1998. Numerical experiments on the residence time distributions of heterogeneous groundwatersheds. Journal of Hydrology, 207(12): 1-17. DOI:http://dx.doi.org/10.1016/S0022-1694(98)00112-7

Małoszewski, P., Zuber, A., 1982. Determining the turnover time of groundwater systems with the aid of environmental tracers: 1 . Models and their applicability. Journal of Hydrology, 57(3-4): 207-231. DOI:http://dx.doi.org/10.1016/00221694(82)90147-0 
Manning, A.H., Caine, J.S., 2007. Groundwater noble gas, age, and temperature signatures in an Alpine watershed: Valuable tools in conceptual model development. Water Resources Research, 43(4): n/a-n/a.

DOI:http://dx.doi.org/10.1029/2006wr005349

Marçais, J., de Dreuzy, J.R., Ginn, T.R., Rousseau-Gueutin, P., Leray, S., 2015. Inferring transit time distributions from atmospheric tracer data: Assessment of the predictive capacities of Lumped Parameter Models on a 3D crystalline aquifer model. Journal of Hydrology, 525(0): 619-631. DOI:http://dx.doi.org/10.1016/j.jhydrol.2015.03.055

Martin, A.D., 2000. Interpretation of residence time distribution data. Chemical Engineering Science, 55(23): 5907-5917. DOI:http://dx.doi.org/10.1016/S00092509(00)00108-1

Massoudieh, A., 2013. Inference of long-term groundwater flow transience using environmental tracers: A theoretical approach. Water Resources Research, 49(12): 8039-8052. DOI:10.1002/2013wr014548

Massoudieh, A., Leray, S., de Dreuzy, J.-R., 2014. Assessment of the value of groundwater age time-series for characterizing complex steady-state flow systems using a Bayesian approach. Applied Geochemistry, 50: 240-251. DOI:http://dx.doi.org/10.1016/j.apgeochem.2013.10.006

Massoudieh, A., Sharifi, S., Solomon, D.K., 2012. Bayesian evaluation of groundwater age distribution using radioactive tracers and anthropogenic chemicals. Water Resources Research, 48(9): n/a-n/a. DOI:http://dx.doi.org/10.1029/2012wr011815

Maxwell, R.M., Welty, C., Tompson, A.F.B., 2003. Streamline-based simulation of virus transport resulting from long term artificial recharge in a heterogeneous aquifer. Advances in Water Resources, 26(10): 1075-1096. DOI:http://dx.doi.org/10.1016/S0309-1708(03)00074-5

McCallum, J.L., Engdahl, N.B., Ginn, T.R., Cook, P.G., 2014. Nonparametric estimation of groundwater residence time distributions: What can environmental tracer data tell us about groundwater residence time? Water Resources Research, 50(3): 2022-2038. DOI:http://dx.doi.org/10.1002/2013wr014974

McDonnell, J.J., Beven, K., 2014. Debates - The future of hydrological sciences: A (common) path forward? A call to action aimed at understanding velocities, celerities and residence time distributions of the headwater hydrograph. Water Resources Research, 50(6): 5342-5350.

DOI:http://dx.doi.org/10.1002/2013WR015141 
McDonnell, J.J. et al., 2010. How old is streamwater? Open questions in catchment transit time conceptualization, modelling and analysis. Hydrological Processes, 24(12): 1745-1754. DOI:http://dx.doi.org/10.1002/hyp.7796

McGuire, K.J., McDonnell, J.J., 2006. A review and evaluation of catchment transit time modeling. Journal of Hydrology, 330(3-4): 543-563.

DOI:http://dx.doi.org/10.1016/j.jhydrol.2006.04.020

McGuire, K.J. et al., 2005. The role of topography on catchment-scale water residence time. Water Resources Research, 41(5): n/a-n/a.

DOI:http://dx.doi.org/10.1029/2004wr003657

Nauman, E.B., 2008. Residence Time Theory. Industrial \& Engineering Chemistry Research, 47(10): 3752-3766. DOI:http://dx.doi.org/10.1021/ie071635a

Neuman, S.P., Tartakovsky, D.M., 2009. Perspective on theories of non-Fickian transport in heterogeneous media. Advances in Water Resources, 32(5): 670-680. DOI:http://dx.doi.org/10.1016/j.advwatres.2008.08.005

Neumann, R.B., LaBolle, E.M., Harvey, C.F., 2008. The Effects of Dual-Domain Mass Transfer on the Tritium-Helium-3 Dating Method. Environmental Science \& Technology, 42(13): 4837-4843. DOI:http://dx.doi.org/10.1021/es7025246

Newman, B.D. et al., 2010. Dating of 'young' groundwaters using environmental tracers: advantages, applications, and research needs. Isotopes in Environmental and Health Studies, 46(3): 259-278.

DOI:http://dx.doi.org/10.1080/10256016.2010.514339

Nir, A., 1964. On the interpretation of tritium 'age' measurements of groundwater. Journal of Geophysical Research, 69(12): 2589-2595. DOI:http://dx.doi.org/10.1029/JZ069i012p02589

Nir, A., 1973. Tracer relations in mixed lakes in non-steady state. Journal of Hydrology, 19(1): 33-41. DOI:http://dx.doi.org/10.1016/0022-1694(73)90091-7

Osenbrück, K. et al., 2006. Timescales and development of groundwater pollution by nitrate in drinking water wells of the Jahna-Aue, Saxonia, Germany. Water Resources Research, 42(12): n/a-n/a. DOI:http://dx.doi.org/10.1029/2006wr004977

Ozyurt, N.N., Bayari, C.S., 2005. LUMPED Unsteady: a Visual Basic® code of unsteady-state lumped-parameter models for mean residence time analyses of groundwater systems. Computers \& Geosciences, 31(3): 329-341.

DOI:http://dx.doi.org/10.1016/j.cageo.2004.09.024

Steady-state analytical RTDs

Page 59 of 77 
Parker, J.C., van Genuchten, M.T., 1984. Flux-Averaged and Volume-Averaged Concentrations in Continuum Approaches to Solute Transport. Water Resources Research, 20(7): 866-872. DOI:http://dx.doi.org/10.1029/WR020i007p00866

Payn, R.A. et al., 2008. Comparison of instantaneous and constant-rate stream tracer experiments through non-parametric analysis of residence time distributions. Water Resources Research, 44(6): n/a-n/a. DOI:http://dx.doi.org/10.1029/2007wr006274

Ponsin, V., Coulomb, B., Guelorget, Y., Maier, J., Höhener, P., 2014. In situ biostimulation of petroleum hydrocarbon degradation by nitrate and phosphate injection using a dipole well configuration. Journal of contaminant hydrology, 171: 22-31. DOI:http://dx.doi.org/10.1016/j.jconhyd.2014.10.003

Press, W.H., 2007. Numerical Recipes 3rd Edition: The Art of Scientific Computing. Cambridge University Press.

Queloz, P. et al., 2015. Transport of fluorobenzoate tracers in a vegetated hydrologic control volume: 2 . Theoretical inferences and modeling. Water Resources Research, 51(4): 2793-2806. DOI:10.1002/2014wr016508

Quintard, M., Whitaker, S., 1993. Transport in ordered and disordered porous media: volume-averaged equations, closure problems, and comparison with experiment. Chemical Engineering Science, 48(14): 2537-2564. DOI:http://dx.doi.org/10.1016/0009-2509(93)80266-S

Raats, P.A.C., 1974. Steady flow patterns in saturated and unsaturated, isotropic soils. Journal of Hydrology, 21(4): 357-369. DOI:http://dx.doi.org/10.1016/S0022$\underline{1694(74) 80005-3}$

Raats, P.A.C., 1977a. Convective transport of solutes by steady flows I. General theory. Agricultural Water Management, 1(3): 201-218. DOI:http://dx.doi.org/10.1016/0378-3774(77)90001-4

Raats, P.A.C., 1977b. Convective transport of solutes by steady flows II. Specific flow problems. Agricultural Water Management, 1(3): 219-232. DOI:http://dx.doi.org/10.1016/0378-3774(77)90002-6

Rinaldo, A. et al., 2015. Storage selection functions: A coherent framework for quantifying how catchments store and release water and solutes. Water Resources Research, 51(6): 4840-4847. DOI:10.1002/2015wr017273

Russian, A., Dentz, M., Le Borgne, T., Carrera, J., Jimenez-Martinez, J., 2013. Temporal scaling of groundwater discharge in dual and multicontinuum catchment models. Water Resources Research, 49(12): 8552-8564. DOI:10.1002/2013wr014255

Steady-state analytical RTDs 
Sanford, W., 2011. Calibration of models using groundwater age. Hydrogeol J, 19(1): 1316. DOI:http://dx.doi.org/10.1007/s10040-010-0637-6

Scher, H., Margolin, G., Metzler, R., Klafter, J., Berkowitz, B., 2002. The dynamical foundation of fractal stream chemistry: The origin of extremely long retention times. Geophysical Research Letters, 29(5): 5-1-5-4.

DOI:http://dx.doi.org/10.1029/2001g1014123

Schumer, R., Benson, D.A., Meerschaert, M.M., Baeumer, B., 2003. Fractal mobile/immobile solute transport. Water Resources Research, 39(10): n/a-n/a. DOI:10.1029/2003wr002141

Schwartz, F.W. et al., 2010. Ambiguous Hydraulic Heads and 14C Activities in Transient Regional Flow. Ground Water, 48(3): 366-379. DOI:10.1111/j.17456584.2009.00655.x

Schwientek, M., Maloszewski, P., Einsiedl, F., 2009. Effect of the unsaturated zone thickness on the distribution of water mean transit times in a porous aquifer. Journal of Hydrology, 373(3-4): 516-526. DOI:http://dx.doi.org/10.1016/j.jhydrol.2009.05.015

Seeboonruang, U., Ginn, T.R., 2006. Upscaling heterogeneity in aquifer reactivity via exposure-time concept: Forward model. Journal of contaminant hydrology, 84(34): 127-154. DOI:http://dx.doi.org/10.1016/j.jconhyd.2005.12.011

Sheahan, N.T., 1977. Injection/Extraction Well System-A Unique Seawater Intrusion Barriera. Ground Water, 15(1): 32-50. DOI:http://dx.doi.org/10.1111/j.1745$\underline{6584.1977 . t b 03150 . x}$

Silva, O. et al., 2009. A general real-time formulation for multi-rate mass transfer problems. Hydrol. Earth Syst. Sci., 13(8): 1399-1411. DOI:10.5194/hess-131399-2009

Solomon, D.K., Genereux, D.P., Plummer, L.N., Busenberg, E., 2010. Testing mixing models of old and young groundwater in a tropical lowland rain forest with environmental tracers. Water Resources Research, 46(4): n/a-n/a. DOI:http://dx.doi.org/10.1029/2009wr008341

Soltani, S.S., Cvetkovic, V., 2013. On the distribution of water age along hydrological pathways with transient flow. Water Resources Research, 49(9): 5238-5245. DOI:10.1002/wrcr.20402

Stolp, B.J. et al., 2010. Age dating base flow at springs and gaining streams using helium-3 and tritium: Fischa-Dagnitz system, southern Vienna Basin, Austria. Water Resources Research, 46(7): n/a-n/a. DOI:http://dx.doi.org/10.1029/2009wr008006

Steady-state analytical RTDs 
Suckow, A., 2012. Lumpy-an interactive lumped parameter modeling code based on MS access and MS excel.

Suckow, A., 2014. The age of groundwater - Definitions, models and why we do not need this term. Applied Geochemistry, 50: 222-230.

DOI:http://dx.doi.org/10.1016/j.apgeochem.2014.04.016

Tang, G., Watson, D.B., Parker, J.C., Brooks, S.C., 2012. A Spreadsheet Program for Two-Well Tracer Test Data Analysis. Groundwater, 50(4): 614-620. DOI:http://dx.doi.org/10.1111/j.1745-6584.2011.00841.x

Tompson, A.F.B., Carle, S.F., Rosenberg, N.D., Maxwell, R.M., 1999. Analysis of groundwater migration from artificial recharge in a large urban aquifer: A simulation perspective. Water Resources Research, 35(10): 2981-2998. DOI:http://dx.doi.org/10.1029/1999WR900175

Turnadge, C., Smerdon, B.D., 2014. A review of methods for modelling environmental tracers in groundwater: Advantages of tracer concentration simulation. Journal of Hydrology, 519, Part D: 3674-3689.

DOI:http://dx.doi.org/10.1016/j.jhydrol.2014.10.056

Turner, J.V., Barnes, C.J., 1998. Modeling of isotope and hydrogeochemical responses in catchment hydrology. Elsevier: Amsterdam.

van der Velde, Y., Torfs, P.J.J.F., van der Zee, S.E.A.T.M., Uijlenhoet, R., 2012. Quantifying catchment-scale mixing and its effect on time-varying travel time distributions. Water Resources Research, 48(6): n/a-n/a.

DOI:http://dx.doi.org/10.1029/2011WR011310

Varni, M., Carrera, J., 1998. Simulation of groundwater age distributions. Water Resources Research, 34(12): 3271-3281. DOI:http://dx.doi.org/10.1029/98wr02536

Visser, A., Broers, H.P., Purtschert, R., Sültenfuß, J., de Jonge, M., 2013. Groundwater age distributions at a public drinking water supply well field derived from multiple age tracers $(85 \mathrm{Kr}, 3 \mathrm{H} / 3 \mathrm{He}$, and 39Ar). Water Resources Research, 49(11): 7778-7796. DOI:http://dx.doi.org/10.1002/2013wr014012

Vogel, J.C., 1967. Investigation of groundwater flow with radiocarbon. In: IAEA (Ed.), Isotopes in Hydrology. IAEA, Vienna, pp. 15.

von Buttlar, H., Libby, W.F., 1955. Natural distribution of cosmic-ray produced tritium. II. Journal of Inorganic and Nuclear Chemistry, 1(1-2): 75-91. DOI:http://dx.doi.org/10.1016/0022-1902(55)80070-X 
Weissmann, G.S., Zhang, Y., LaBolle, E.M., Fogg, G.E., 2002. Dispersion of groundwater age in an alluvial aquifer system. Water Resources Research, 38(10): 16-1. DOI:http://dx.doi.org/10.1029/2001WR000907

Wiener, N., 1949. Extrapolation, interpolation, and smoothing of stationary time series, 2. MIT press Cambridge, MA.

Wolf, D., Resnick, W., 1963. Residence Time Distribution in Real Systems. Industrial \& Engineering Chemistry Fundamentals, 2(4): 287-293.

DOI:http://dx.doi.org/10.1021/i160008a008

Zhang, Y., Baeumer, B., Benson, D.A., 2006. Relationship between flux and resident concentrations for anomalous dispersion. Geophysical Research Letters, 33(18): n/a-n/a. DOI:http://dx.doi.org/10.1029/2006gl027251

Zouari, K., Trabelsi, R., Chkir, N., 2011. Using geochemical indicators to investigate groundwater mixing and residence time in the aquifer system of Djeffara of Medenine (southeastern Tunisia). Hydrogeol J, 19(1): 209-219.

DOI:http://dx.doi.org/10.1007/s10040-010-0673-2

Zuber, A., Różański, K., Kania, J., Purtschert, R., 2011. On some methodological problems in the use of environmental tracers to estimate hydrogeologic parameters and to calibrate flow and transport models. Hydrogeol J, 19(1): 53-69. DOI:http://dx.doi.org/10.1007/s10040-010-0655-4 


\section{FIGURES}

Figure 1: Conceptual sketch of RTDs generation. The RTD is defined for every single point in the system. The type of sampling (e.g. localized, spread, at the exit) directly affects the form of the RTD: the more it catches flow paths, the spreader the distribution is. Despite that, the details of the RTD and of the flow paths caught by the sampling cannot be identified.

Figure 2: Residence Time Distributions $\rho_{T}(a)$ for globally mixed systems as a function of the normalized residence time $a / \tau$. Phenomena leading to a deviation from perfect mixing are illustrated. The RTDs share the same $\beta$.

Figure 3: Physical configurations yielding an analytical solution for the exit RTD $\rho_{T}(a)$ in the absence of diffusion and hydrodynamic dispersion. Unless otherwise specified, RTDs are displayed as a function of the normalized residence time $a / \tau$. Equations for the RTD and the mean residence time are given in the text as indicated in the third column. All boundaries are no-flow unless indicated. a) and e) the RTD stops at 1 for better visualization. b) The cases of partial sampling for which either the upper or lower part of the aquifer is not sampled are displayed for $C_{N S}=0.25$. c) and d) To directly compare the distributions for various ratios $R_{L} / R_{0}$ or $H_{L} / H_{0}$, the RTD is multiplied by the mean residence time $\tau$. In such a way, the area under the curve is always 1 and the proportion of each time bin is readily accessible. f) The residence time is normalized here by its median value $a / a_{c}$. The first arrival time is at $\frac{a_{c}}{3}$. At late-time, the RTD has a power law behavior with a slope of $-\frac{4}{3}$ (dash-dot gray line).

Figure 4: Conceptual sketch of a 1-D horizontal aquifer under uniform recharge conditions. The portion of non-sampled flow lines $C_{N S}$ with a partially-penetrating well is either defined by $H_{u p} / H_{H}$ or $H_{\text {low }} /_{H}$. No-flow boundaries are illustrated by dashes.

Figure 5: Residence Time Distribution $\rho_{T}(a)$ for an advective-dispersive stream tube in flux (outflow) averaged or volume (resident) averaged sampling conditions as a function of the 
normalized residence time $a / \tau$ for various Peclet numbers. The RTDs share the same mean residence time $\tau$.

Figure 6: Conceptual scheme of parallel (a) and series (b) mixing of models.

Figure 7: Residence Time Distribution $\rho_{T}(a)$ of the Gamma model. The RTDs share the same $\varepsilon(=0)$ and $\kappa(=2)$. To directly compare the distributions for various $\alpha$, the RTD is multiplied by the mean residence time $\tau$. In such a way, the area under the curve is always 1 and the proportion of each time bin is readily accessible. The exponential model is plotted in black.

Figure 8: Conceptual schemes of lagged systems. Piston compartment is (a) a vertically leaking compartment which is not sampled or (b) a confined compartment.

Figure 9: Conceptual scheme of the scale-dependent dispersion model. The sampling point (red square) is located at the depth $H_{w}$ below the water table. The longitudinal velocity $v_{l}$ is linked to the horizontal $v_{h}$ and vertical $v_{v}$ velocities simply by $\sqrt{v_{h}^{2}+v_{v}^{2}}$.

Table 1: Shift $a_{0}$ for a few types of adjacent aquifer. $L$ and $L_{d}$ are the extension of the (non-piston) adjacent and (piston) confined compartments respectively. The first example is commonly referred to as the exponential-piston model. 


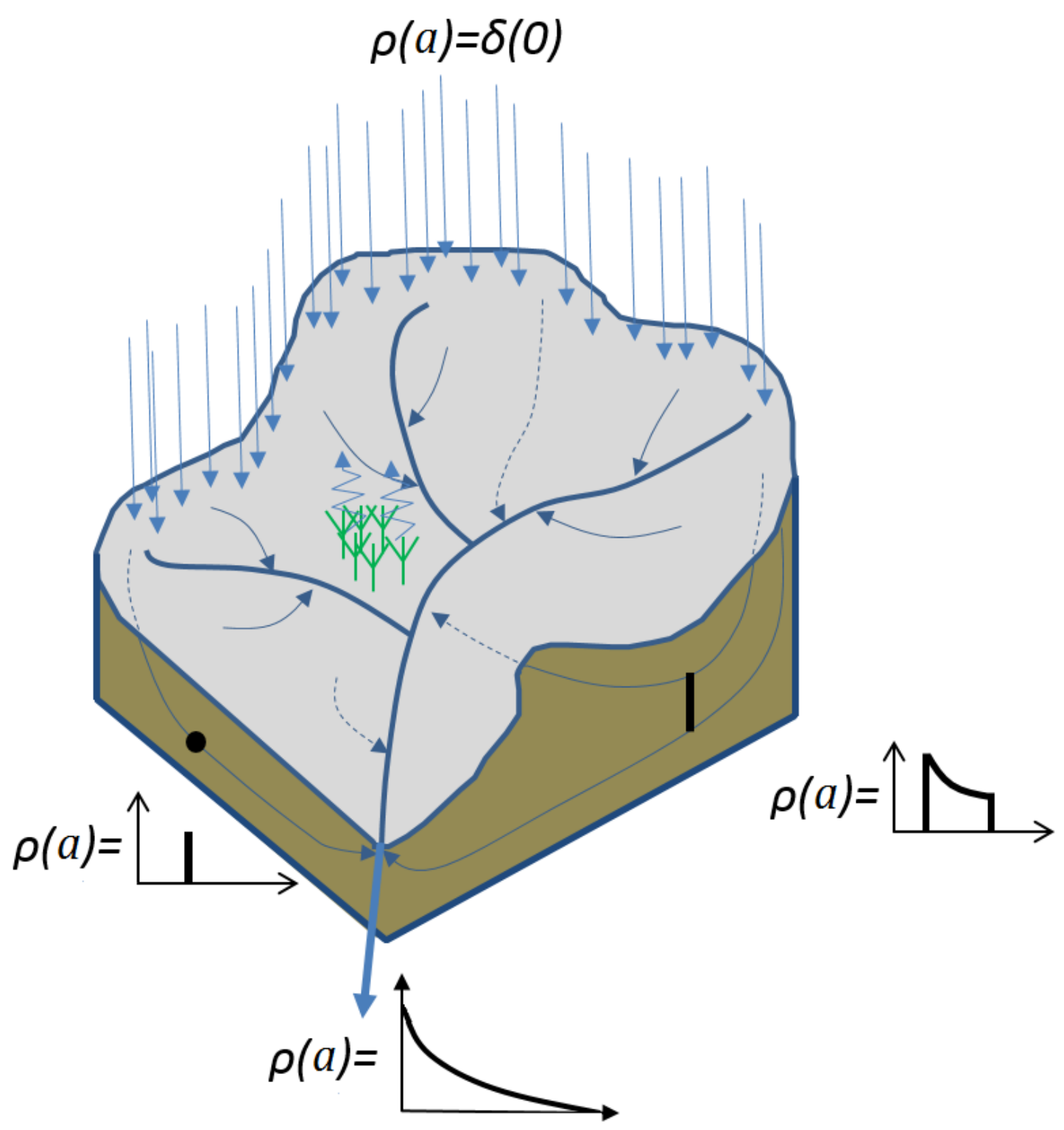




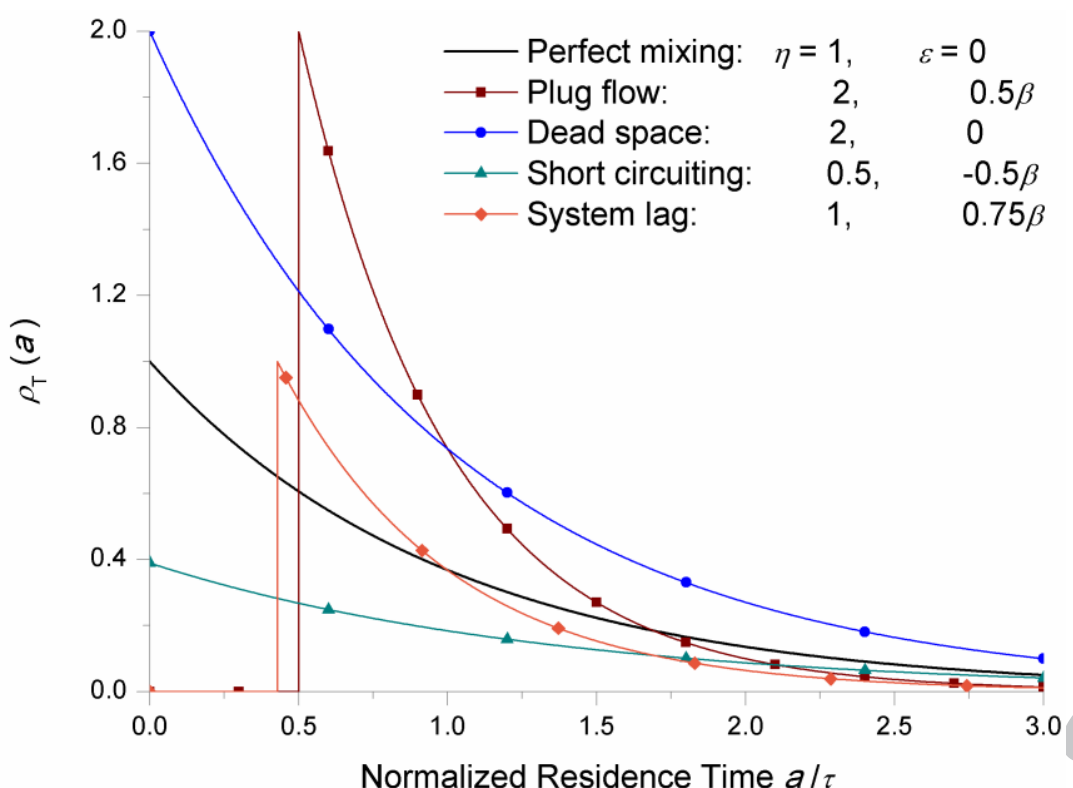

Steady-state analytical RTDs 


\section{ACCEPTED MANUSCRIPT}

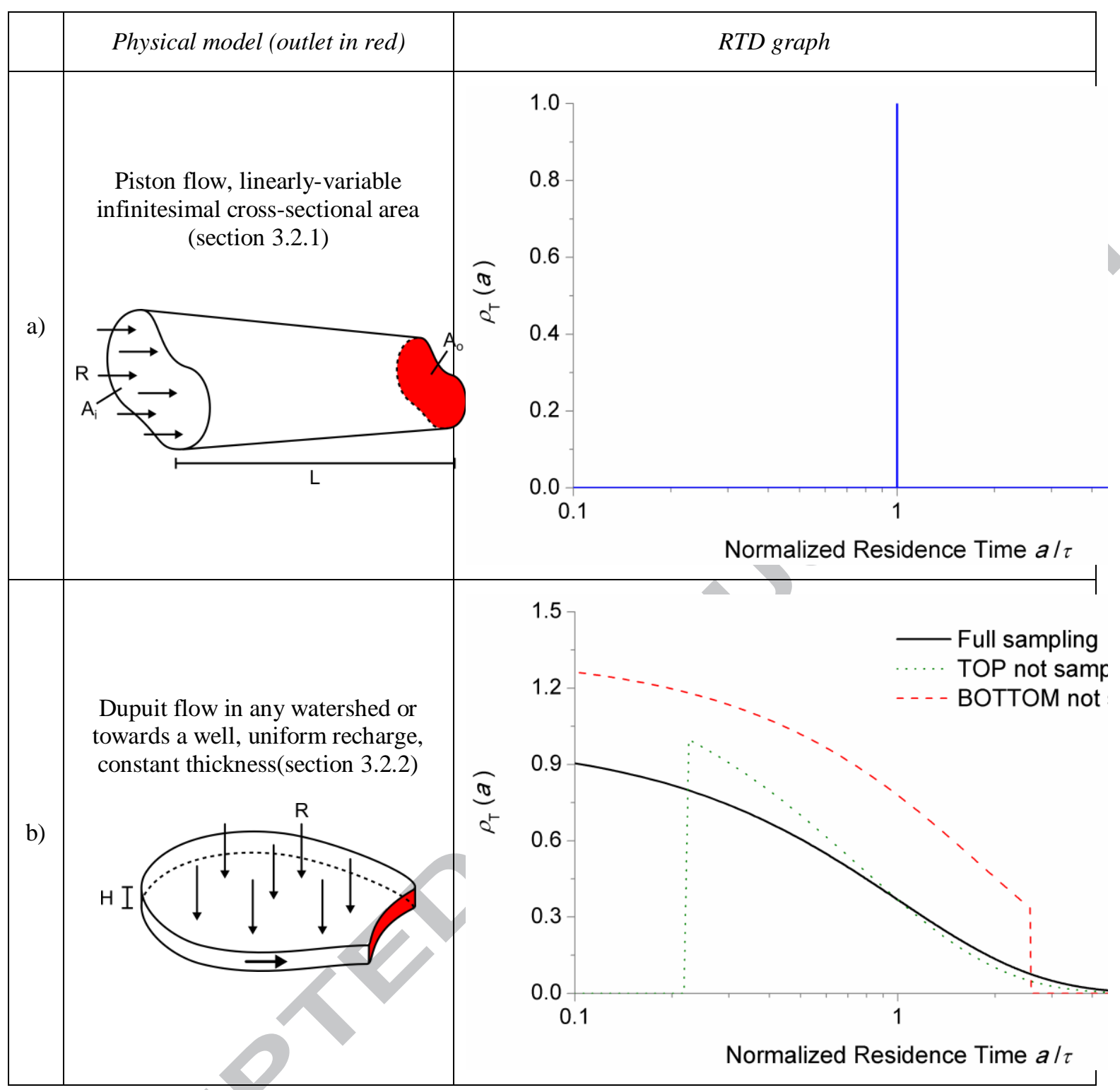

Steady-state analytical RTDs 


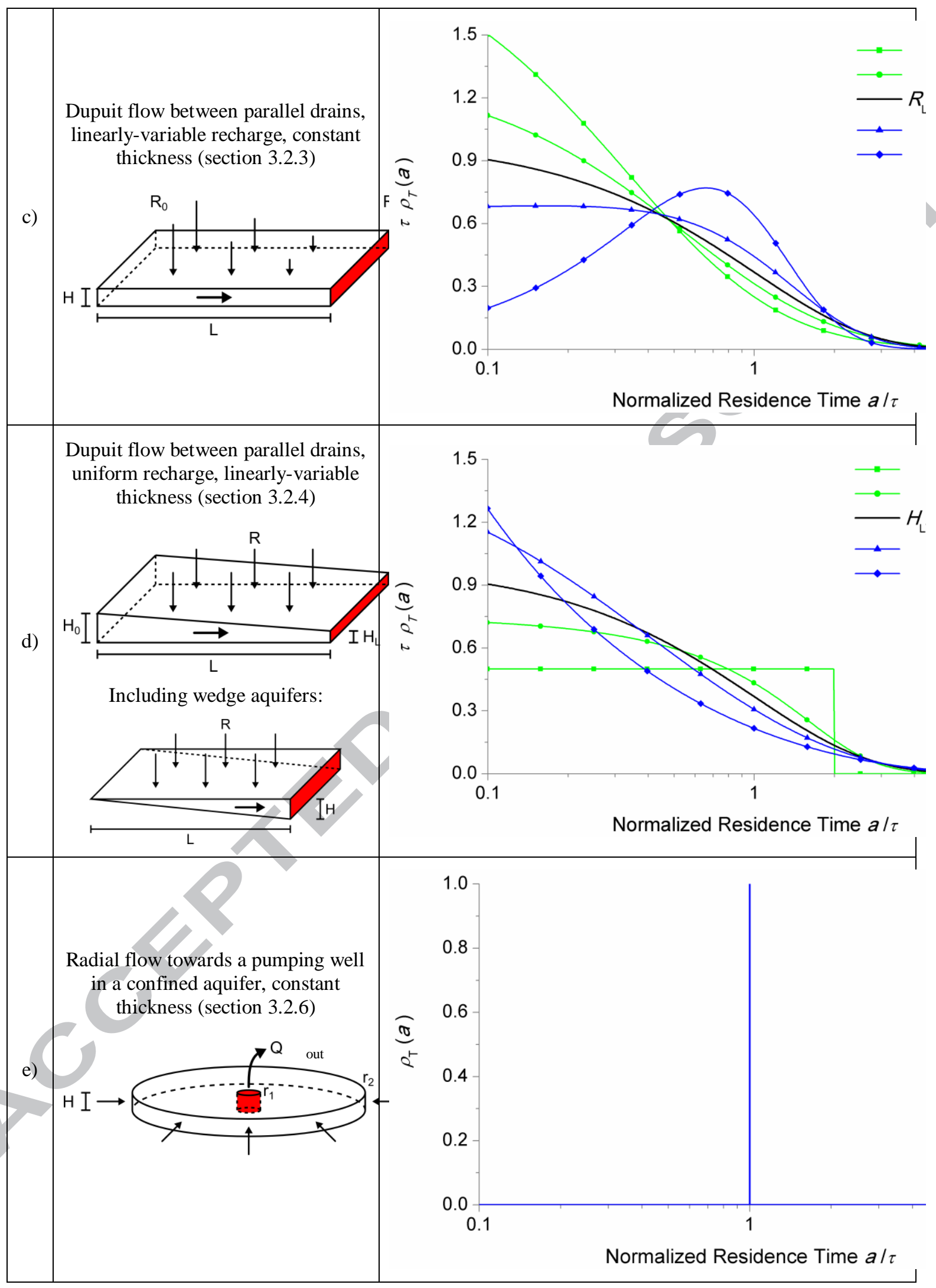

Steady-state analytical RTDs 


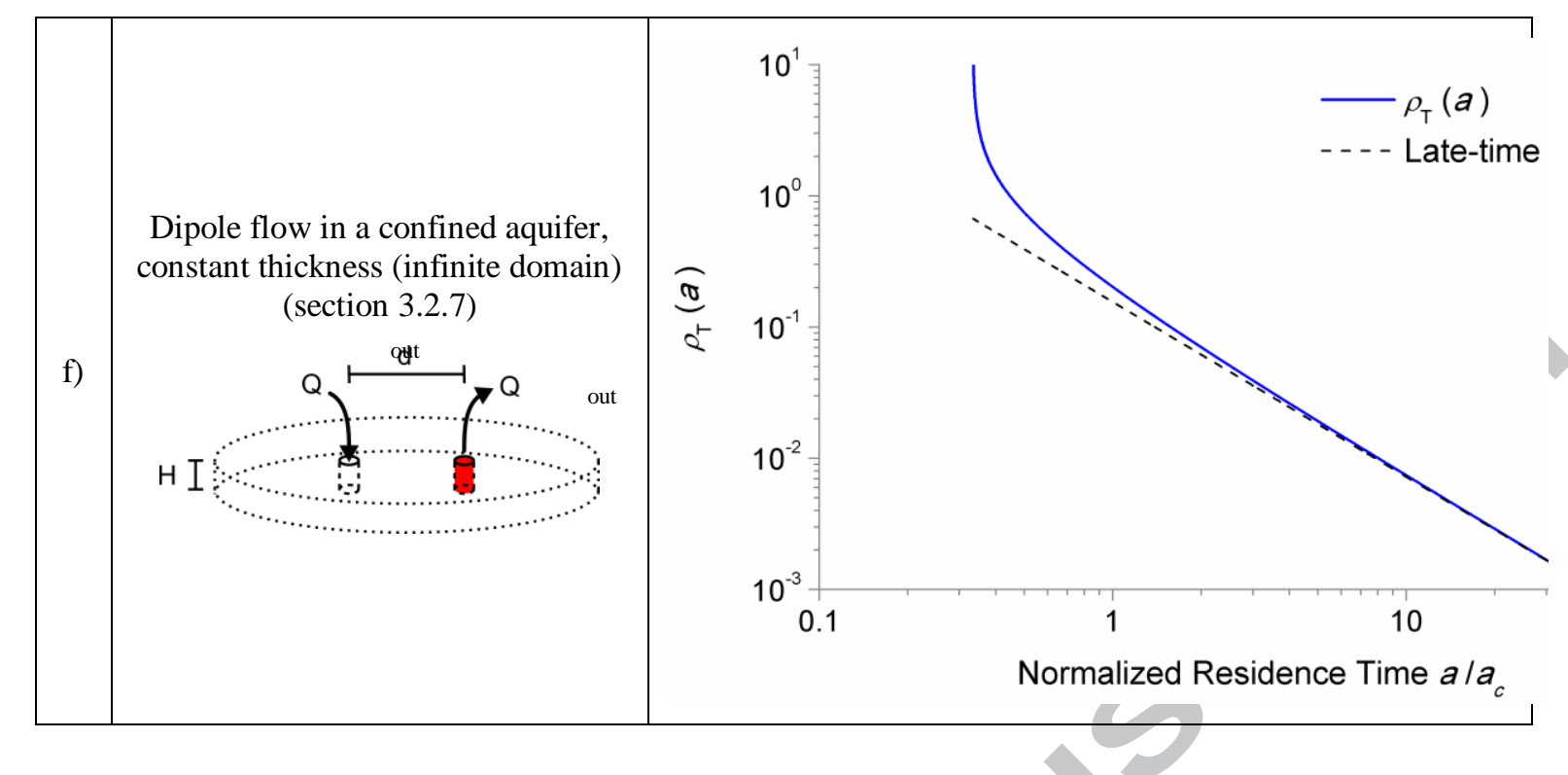

Steady-state analytical RTDs 


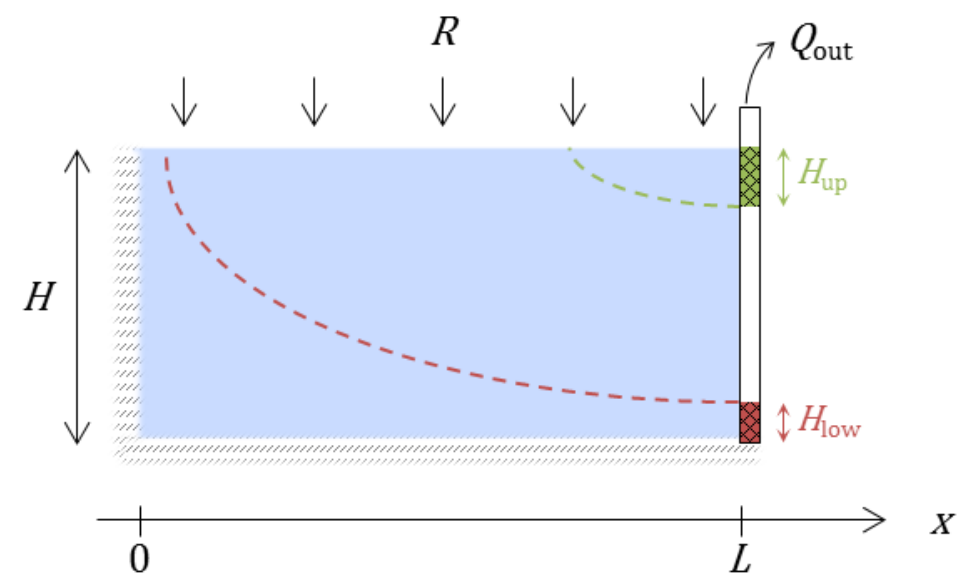




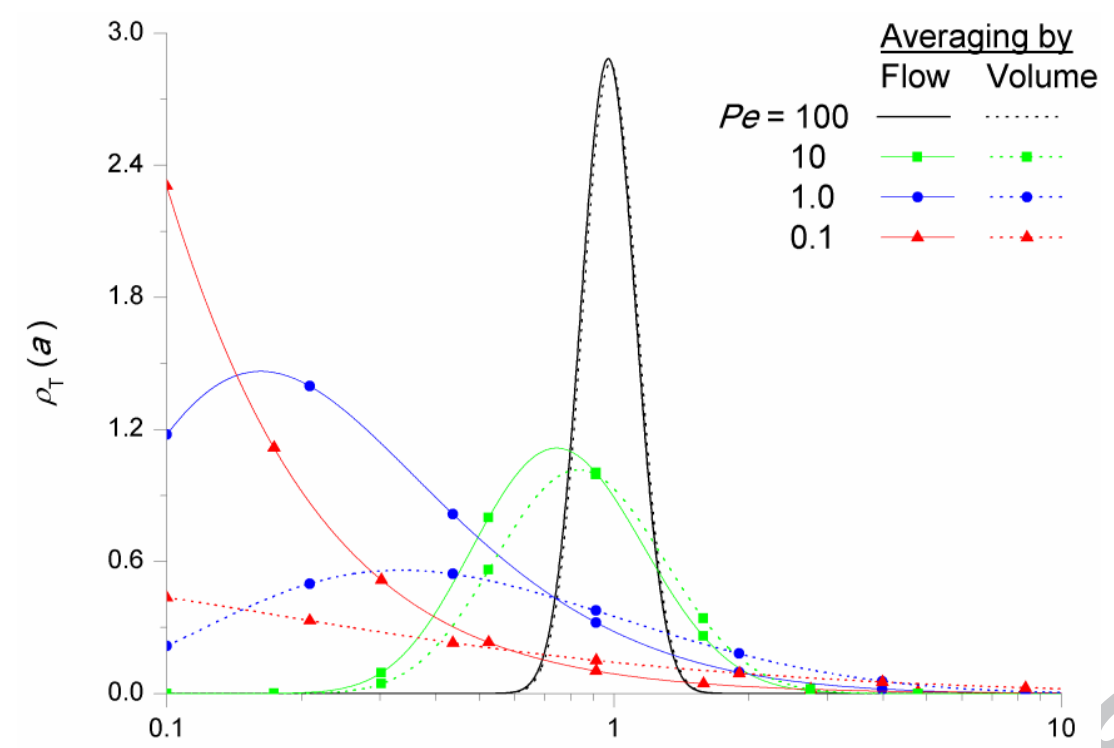

Normalized Residence Time $a / \tau$

Steady-state analytical RTDs 
(a)

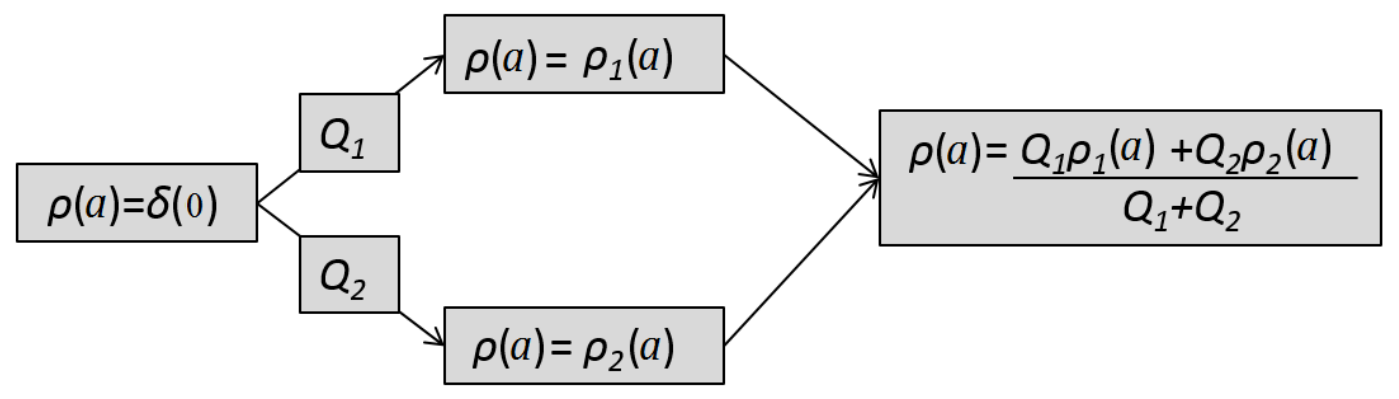

(b)

$\rho(a)=\delta(0) \rightarrow \rho(a)=\rho_{1}(a) \rightarrow \rho(a)=\rho_{2}(a) \rightarrow \rho(a)=\rho_{1}(a) * \rho_{2}(a)$



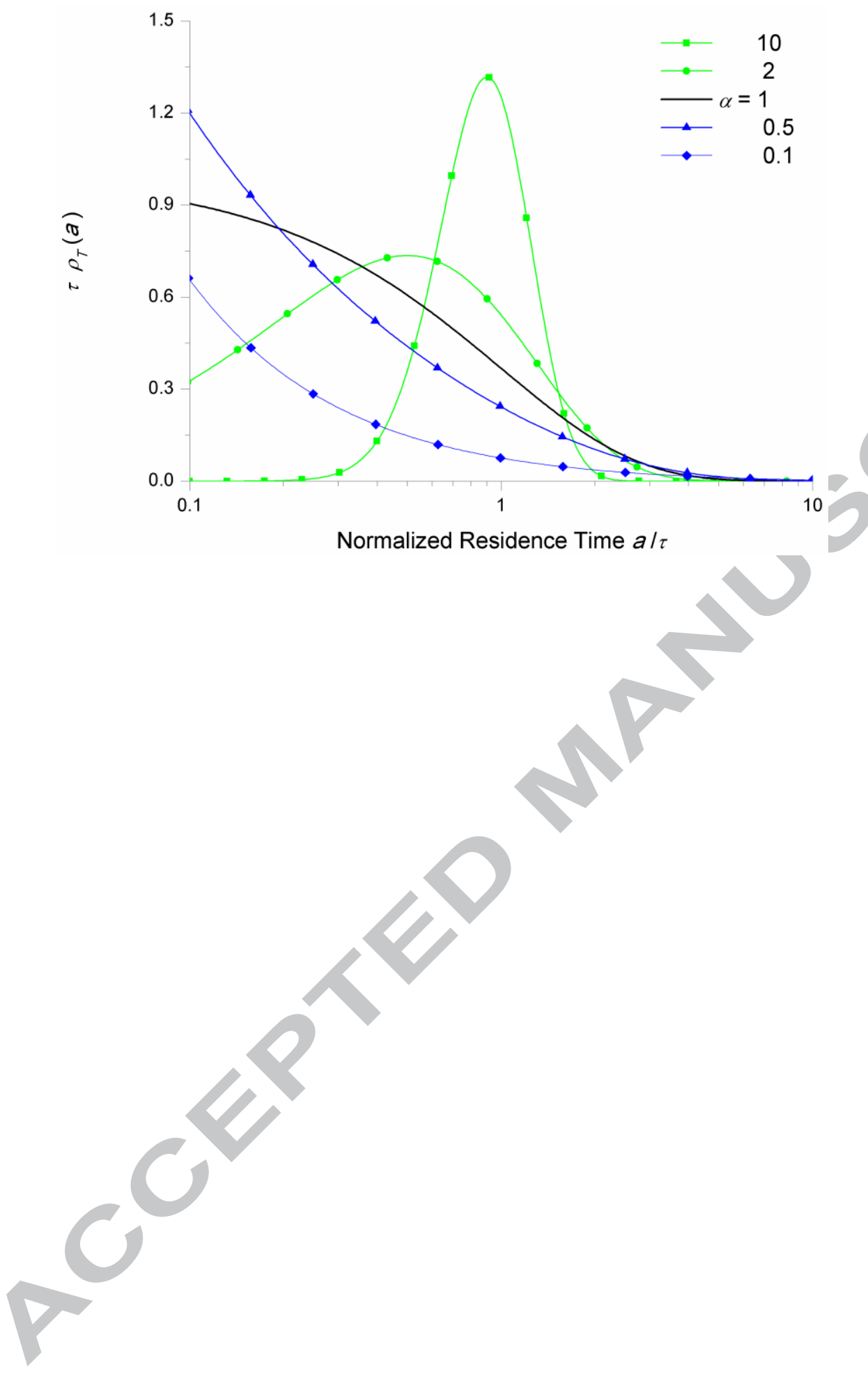

Steady-state analytical RTDs 
(a)

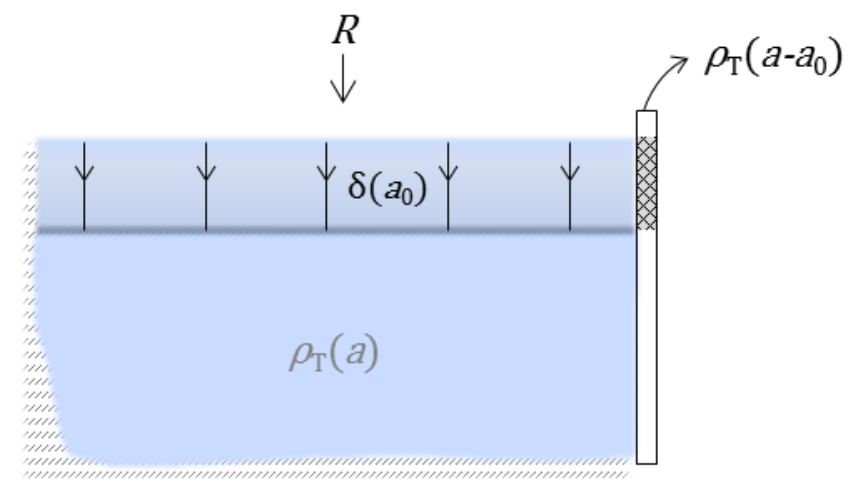

(b)

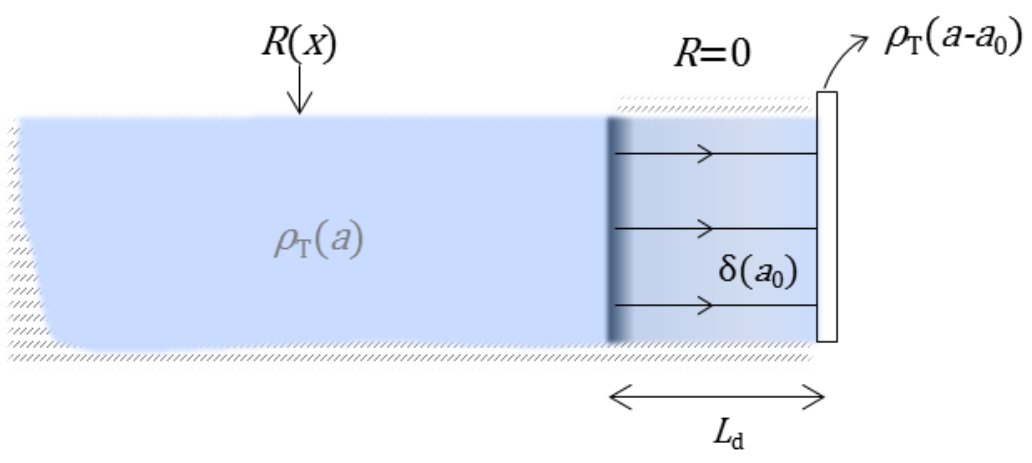



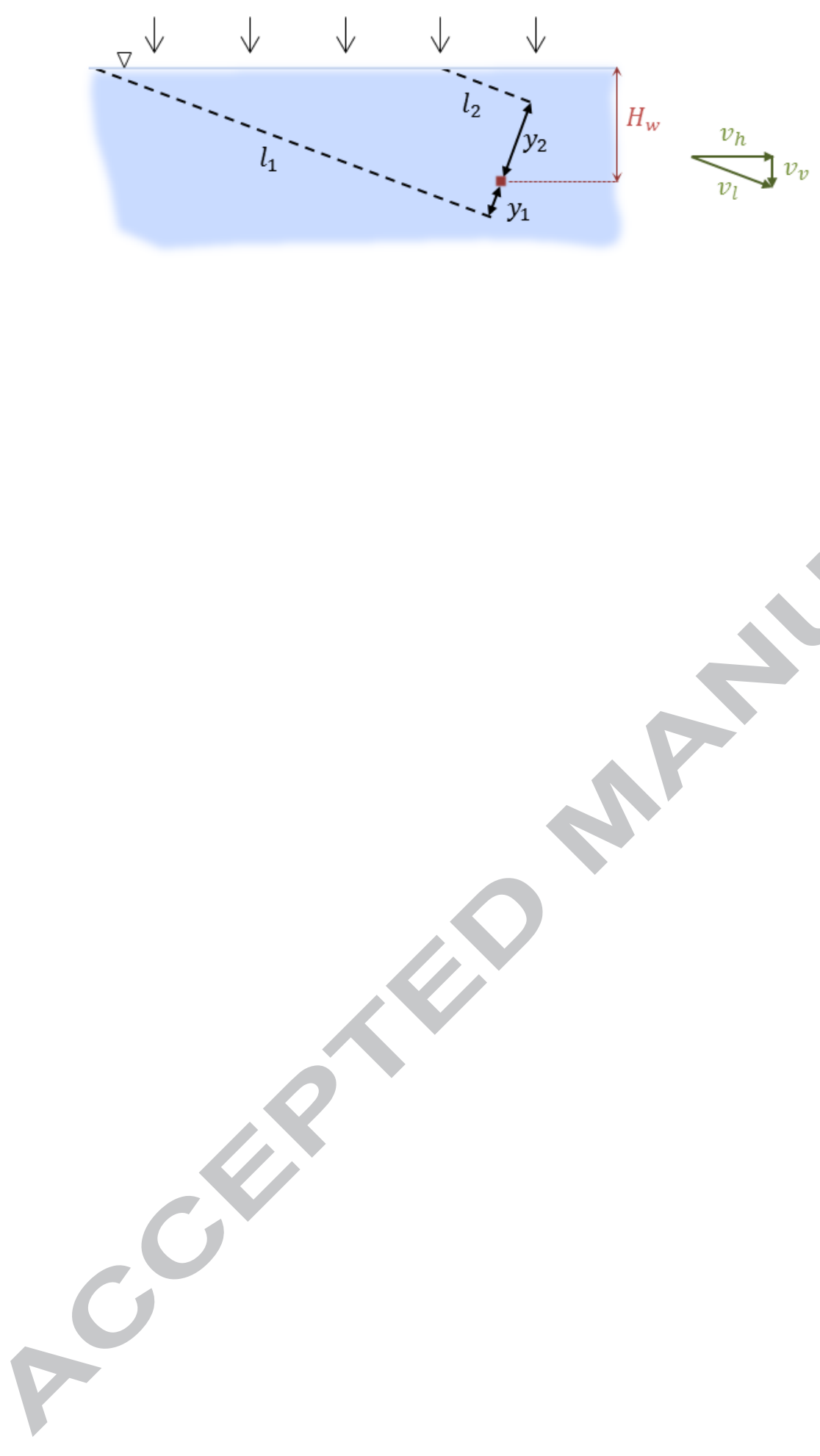

Steady-state analytical RTDs 


\begin{tabular}{|l|c|c|}
\hline Adjacent aquifer & $\begin{array}{l}\text { Velocity at adjacent aquifer } \\
\text { exit }\end{array}$ & Shift $a_{0}$ \\
\hline $\begin{array}{l}\text { Thickness } H \text { and recharge } R \text { constant (see } \\
\text { 3.2.2) }\end{array}$ & $\frac{R L}{H}$ & $\frac{\theta H L_{d}}{R L}$ \\
\hline $\begin{array}{l}\text { Thickness } H \text { constant and recharge } R \\
\text { variable (see 3.2.3) }\end{array}$ & $\frac{R_{0}+R_{L}}{2} \frac{L}{H}$ & $\frac{2 \theta H L_{d}}{\left(R_{0}+R_{L}\right) L}$ \\
\hline $\begin{array}{l}\text { Thickness } H \text { variable and recharge } R \\
\text { constant (see 3.2.4) }\end{array}$ & $\frac{R L}{H_{L}}$ & $\frac{\theta H_{L} L_{d}}{R L}$ \\
\hline
\end{tabular}




\section{Highlights}

- Steady-state analytical solutions for residence time distributions are reviewed

- Analytical solutions assume simple and parameterizable physical configurations

- Physically-based solutions are a powerful tool for understanding hydrologic systems

- They are to be used with careful account of the conditions for their relevance

- Alternative methods (e.g. shape-free distributions) exist for more complex systems 